April 28, 2007

\title{
Distributive lattices defined for representations of rank two semisimple Lie algebras
}

\author{
L. Wyatt Alverson $\mathrm{II}^{1}$, Robert G. Donnelly ${ }^{1}$, Scott J. Lewis ${ }^{1}$, Marti McClard ${ }^{2}$, Robert Pervine ${ }^{1}$, \\ Robert A. Proctor ${ }^{3}$, N. J. Wildberger ${ }^{4}$ \\ ${ }^{1}$ Department of Mathematics and Statistics, Murray State University, Murray, KY 42071 \\ ${ }^{2}$ Department of Mathematics, University of Tennessee, Knoxville, TN 37996 \\ ${ }^{3}$ Department of Mathematics, University of North Carolina, Chapel Hill, NC 27599 \\ ${ }^{4}$ School of Mathematics, University of New South Wales, Sydney, NSW 2052
}

\begin{abstract}
For a rank two root system and a pair of nonnegative integers, using only elementary combinatorics we construct two posets. The constructions are uniform across the root systems $A_{1} \oplus A_{1}, A_{2}, C_{2}$, and $G_{2}$. Examples appear in Figures 3.2 and 3.3. We then form the distributive lattices of order ideals of these posets. Corollary 5.4 gives elegant quotient-of-products expressions for the rank generating functions of these lattices (thereby providing answers to a 1979 question of Stanley). Also, Theorem 5.3 describes how these lattices provide a new combinatorial setting for the Weyl characters of representations of rank two semisimple Lie algebras. Most of these lattices are new; the rest of them (or related structures) have arisen in work of Stanley, Kashiwara, Nakashima, Littelmann, and Molev. In a future paper, one author shows that the posets constructed here form a Dynkin diagram-indexed answer to a combinatorially posed classification question. In a companion paper, some of these lattices are used to explicitly construct some representations of rank two semisimple Lie algebras. This implies that these lattices are strongly Sperner.
\end{abstract}

Keywords. Distributive lattice, rank generating function, rank two semisimple Lie algebra, representation AMS subject classifications. 05A15, 05E10, 17B10

\section{Introduction}

One of the earliest combinatorial forays into Lie representation theory was Stanley's [Sta1] in 1979. Certain polynomials arising from representations which had elegant quotient-of-product forms captured his attention. He observed that some of these polynomials were the rank generating functions of certain distributive lattices. In Problem 3 of [Sta1] he asked if further distributive lattices could be found which would be associated to more of the polynomials. Consider the poset " $\mathbf{2} \times \mathbf{3}$ " shown in Figure 1.1, the product of chains with 2 and 3 elements. Its lattice $L(2,3)=J(\mathbf{2} \times \mathbf{3})$ of order ideals is shown in Figure 1.1. Stanley knew that the rank generating function for the general case $L(k, n+1-k)=J(\mathbf{k} \times(\mathbf{n}+\mathbf{1}-\mathbf{k}))$ satisfies the identity

$$
\sum N_{j} q^{j}=\frac{\left(1-q^{n+1}\right)\left(1-q^{n}\right) \cdots\left(1-q^{n+2-k}\right)}{\left(1-q^{k}\right)\left(1-q^{k-1}\right) \cdots(1-q)}
$$

where $N_{j}$ is the number of order ideals in $\mathbf{k} \times(\mathbf{n}+\mathbf{1}-\mathbf{k})$ with $j$ elements. The right hand side is the "Gaussian coefficient" $q$-analog of the binomial coefficient $\left(\begin{array}{c}n+1 \\ k\end{array}\right)$. It is also a shifted version of the principal specialization of the Weyl character for the $k$ th fundamental representation of the Lie algebra $\mathfrak{s l}(n+1, \mathbb{C})$, the rank $n$ simple Lie algebra of type $A$. These considerations led Stanley to introduce the more general distributive lattices $L(\lambda, n+1)$, whose elements are semistandard tableaux of shape $\lambda$ with entries from $\{1,2, \ldots, n+1\}$. Similar identities hold for the 
rank generating functions of these lattices. Stanley was aware that the polynomial associated to the "last" fundamental representation of the Lie algebra $\mathfrak{s p}(2 n, \mathbb{C})$ specializes to the $(n+1)$ st Catalan number $\frac{2}{n+2}\left(\begin{array}{c}2 n+1 \\ n\end{array}\right)$ when $q$ is set to 1 . Thus the principal specialization of the Weyl character for that representation is a $q$-analog to the $(n+1)$ st Catalan number. The second author of this paper constructed a poset $P_{n}$ such that the distributive lattice $L_{n}=J\left(P_{n}\right)$ of its order ideals has rank generating function $\frac{1-q^{2}}{1-q^{n+2}}\left(\begin{array}{c}2 n+1 \\ n\end{array}\right)$, a shifted version of the principal specialization. So the total number of order ideals from $P_{n}$ is the $(n+1)$ st Catalan number. This result now appears as part (ccc) of Exercise 6.19 of [Sta3]. See Figure 1.1 for the poset $P_{3}$; it has 14 order ideals.

Figure 1.1

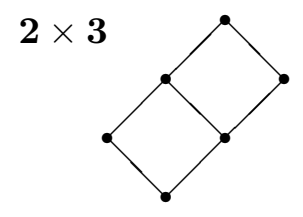

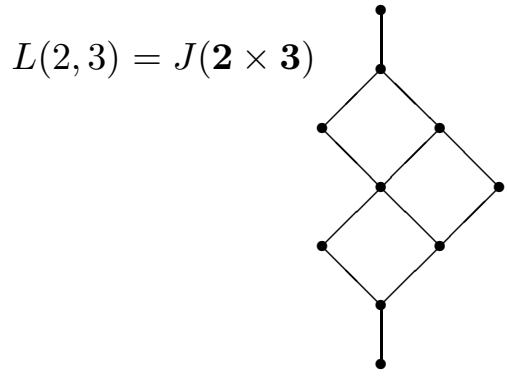

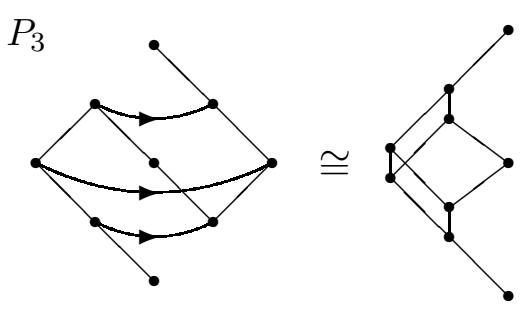

Here is Stanley's 1979 question:

Problem 3: Which other of the polynomials [of Theorem 1] are the rank generating functions for distributive lattices (or perhaps just posets) "naturally associated" with the root system $R$ ?

We supply answers to this question by constructing eight two-parameter families of distributive lattices. By the proof of Corollary 5.4, their rank generating functions are the shifted principal specializations of the Weyl characters of the irreducible finite dimensional representations of the four rank two semisimple Lie algebras $A_{1} \oplus A_{1}, A_{2}, C_{2}$, and $G_{2}$. The answers for $C_{2}$ and $G_{2}$ are largely new. Given a rank two semisimple Lie algebra $\mathfrak{g}$ and a pair of non-negative integers, we first construct two "g-semistandard posets". The "g-semistandard" distributive lattices are then obtained by ordering the order ideals of these posets by inclusion. For example, the choices of $G_{2}$ and non-negative integer parameters $(2,2)$ specify the last poset in each of Figures 3.2 and 3.3. According to Corollary 5.4, both of these posets have $\frac{1}{5 !}(3 \cdot 3 \cdot 6 \cdot 9 \cdot 12 \cdot 15)=729=3^{6}$ order ideals. The rank generating function for both of the corresponding $G_{2}$-semistandard lattices is

$$
R G_{G_{2}}(2,2, q)=\frac{\left(1-q^{3}\right)\left(1-q^{3}\right)\left(1-q^{6}\right)\left(1-q^{9}\right)\left(1-q^{12}\right)\left(1-q^{15}\right)}{(1-q)(1-q)\left(1-q^{2}\right)\left(1-q^{3}\right)\left(1-q^{4}\right)\left(1-q^{5}\right)}
$$

Hence our lattices $L_{G_{2}}^{\beta \alpha}(2,2)$ and $L_{G_{2}}^{\alpha \beta}(2,2)$ are two answers to Problem 3 .

Since the 1970's, the "zoo" of finite sets of combinatorial objects which are enumerated by quotient-of-products formulas has grown to include dozens of species. Here Corollary 5.4 adds $L_{C_{2}}^{\beta \alpha}(a, b), L_{C_{2}}^{\alpha \beta}(a, b), L_{G_{2}}^{\beta \alpha}(a, b)$, and $L_{G_{2}}^{\alpha \beta}(a, b)$ to this zoo; they are analogs to the lattices $L(\lambda, n)$. Our $\mathfrak{g}$-semistandard lattices are uniformly defined across the four types of rank two semisimple Lie algebras. Corollary 5.4 also notes that the sequence of rank cardinalities for any $\mathfrak{g}$-semistandard lattice is symmetric and unimodal. 
Only familiarity with the most basic Lie representation theory in [Hum] is needed to read this paper. The central fact needed is that each irreducible finite dimensional representation of a semisimple Lie algebra of rank $n$ has a unique $n$-variate Weyl character.

Some of the rank two g-semistandard lattices constructed here (or related objects) have appeared in the work of Stanley, Kashiwara, Nakashima, Littelmann, Molev, and several of the authors. However, taken as a whole, each of the $C_{2^{-}}$and $G_{2^{-}}$-families of $\mathfrak{g}$-semistandard lattices is new. The $A_{2}$-family of semistandard lattices here are the $n=2$ case of the $L(\lambda, n)$ lattices introduced in [Sta1]. A certain infinite subfamily of the $C_{2}$-semistandard lattices appeared in [DLP] as the $n=2$ case of the "Molev lattices" $L_{B}^{M o l}(k, 2 n)$. A certain infinite subfamily of the $G_{2}$-semistandard lattices was studied in $[\mathrm{DLP}]$.

Let $\mathfrak{g}$ be a semisimple Lie algebra of rank $n$. Various data and structures have been associated to each irreducible finite dimensional representation of $\mathfrak{g}$, starting with its highest weight and dimension. Once certain subalgebras of $\mathfrak{g}$ have been fixed, the multiset of weights of a representation is determined. The Weyl character of the representation is the generating function for this multiset of weights. It is a Laurent polynomial in $n$ variables. The polynomials that caught Stanley's eye were shifted versions of the "principal specializations" of the Weyl characters to the variable $q$. A finer version of Stanley's 1979 question is: For each Weyl character, find a distributive lattice with weighted vertices such that the sum of these weights is the Weyl character. If the lattice elements are assigned weights in a reasonable manner, then a shifted version of the principal specialization will be the lattice's rank generating function. An explicit combinatorial answer to this question (such as a lattice constructed from tableaux) will include a solution to the "labelling problem" for the character: the lattice elements will be combinatorial objects which can be used as labels for the weights. The problem considered here is a stronger version of this finer version of Stanley's question for $n=2$. The "stronger" aspect is described below.

Going further, fixing Chevalley generators for $\mathfrak{g}$ and basis vectors for the representation space determines the data consisting of the entries of the representing matrices for the generators. At this point in several papers (such as [Don1]), the second author introduces the "supporting graph" combinatorial structure. This is a directed graph whose edges are colored by the simple roots of $\mathfrak{g}$. The edges colored by simple root $\alpha_{i}$ indicate which basis vectors arise with non-zero coefficients when the Chevalley generators $x_{i}$ and $y_{i}$ of $\mathfrak{g}$ act on the various basis vectors. This graph is actually the Hasse diagram of a poset. Several of the authors have been able to find distributive lattice supporting graphs for many representations [Don1], DLP], [ADLP]. The crystal graph is another combinatorial structure associated to a representation. For irreducible representations, the crystal graph is a supporting graph when the weight multiplicities are all one. Such representations have only one supporting graph. But otherwise the crystal graph has fewer edges than do the most efficient supporting graphs; then it cannot support its representation.

Our original goal for developing $\mathfrak{g}$-semisimple lattices was to supply uniformly constructed labels and supporting graphs for explicit realizations of all irreducible representations of any rank two semisimple Lie algebra $\mathfrak{g}$. Suppose a vertex-weighted edge-colored directed graph is proposed to be a supporting graph of a representation of $\mathfrak{g}$ : In addition to its vertex weighting agreeing with the 
Weyl character, its edge-coloring must also satisfy certain conditions specified by the Cartan matrix of $\mathfrak{g}$. (But these conditions alone are not sufficient for the graph to be a supporting graph.) If these edge-coloring necessary conditions are also met, the proposed graph is said to be a "splitting poset" for the representation. The edge-coloring conditions are the embodiment of Stanley's request that the lattices be natural with respect to the Lie theory. Here is the "stronger" aspect of our main problem: Not only do we require that the weighting of their elements agree with a given Weyl character, we seek edge-colored distributive lattices which are splitting posets. Our answer to this question consists of the $\mathfrak{g}$-semistandard lattices: Proposition 4.2 verifies that the edge colorings satisfy the necessary conditions and our main result Theorem 5.3 verifies that the vertex weights agree with the character. (The latter verification implies that the order ideals in the $\mathfrak{g}$-semistandard posets can serve as new weight labels for these representations.)

The necessary edge-color conditions correspond to the Serre relations (S1) and (S3) of Proposition 18.1 of $\mathrm{Hum}$. Given a splitting poset for a representation of $\mathfrak{g}$, if edge coefficients for the actions of the generators $x_{i}$ and $y_{i}$ of $\mathfrak{g}$ can be found that satisfy the relations (S2), then a result of Kashiwara's implies that the remaining Serre relations $\left(\mathrm{S}_{i j}^{+}\right)$and $\left(\mathrm{S}_{i j}^{-}\right)$are automatically satisfied. In certain cases the companion paper $\mathrm{ADLP}$ is able to attain our original goal by assigning coefficients satisfying (S2) to the edges of the lattices introduced here. So [ADLP presents explicit realizations for the following irreducible representations of rank two simple Lie algebras, indexed by their type and highest weights: $A_{2}\left(a \omega_{1}+b \omega_{2}\right), C_{2}\left(a \omega_{1}\right), C_{2}\left(b \omega_{2}\right), C_{2}\left(\omega_{1}+b \omega_{2}\right), G_{2}\left(a \omega_{1}\right), G_{2}\left(\omega_{2}\right)$, for $a, b \geq 0$. Since the $\mathfrak{g}$-semistandard lattices are supporting graphs here, as in [Pr2] they can be seen to be "strongly Sperner". The results of this paper facilitated the new $C_{2}\left(\omega_{1}+b \omega_{2}\right)$ constructions and made it possible to now present the supporting lattices for all of these representations in a uniform fashion.

It can be shown that the $\mathfrak{g}$-semistandard lattices corresponding to the other rank two irreducible representations cannot support their corresponding representations. But to state Corollary 5.4, one only needs to know that the lattice at hand is a splitting poset for an irreducible representation. Hence the beautiful product identities may be written down for the rank generating functions of all $\mathfrak{g}$-semistandard lattices. The necessary edge-coloring conditions are so strong that the second author has been able to prove that the Dynkin diagram-indexed $\mathfrak{g}$-semistandard lattices constitute the entire answer to a purely combinatorial problem [Don2]. See Theorems 6.1 and 6.2.

The positioning of splitting posets (in general; $\mathfrak{g}$-semistandard lattices in particular) in the world of combinatorial structures associated to representations is vaguely similar in spirit to the positioning of crystal graphs: both the lattices and crystal graphs superimpose additional combinatorial structure onto the data contained in the Weyl character, but neither can always support the actions of the corresponding representations. In Section 6 we indicate how some splitting posets may hopefully someday be used instead of crystal graphs for some purposes, such as computing tensor products.

Many of the definitions, lemmas, and propositions developed in this paper are needed in [ADLP]. Some of them will also be used in [DW] to explicitly construct many families of splitting posets for the simple Lie algebras $A_{n}, B_{n}, C_{n}, D_{n}, E_{6}, E_{7}$, and $G_{2}$. 
Section 2 presents definitions and some preliminary and background results. The reader should initially browse this section and then consult it as needed. Section 3 further considers "grid posets" which were introduced in ADLP and whose definition is purely combinatorial. Lemma 3.1 is the key decomposition result. It is proved here and used in [ADLP] and [DW]. Section 4 introduces $\mathfrak{g}$-semistandard posets, $\mathfrak{g}$-semistandard lattices, and $\mathfrak{g}$-semistandard tableaux. Section 5 shows that the elements of these lattices match up with tableaux presented in Littelmann's [Lit]. This match-up yields our main results. Section 6 contains further remarks and problems.

\section{Definitions and preliminary results}

The reference for standard combinatorics material is [Sta2], and the reference for standard representation theory material is [Hum]. We use " $R$ " (and " $Q$ ") as a generic name for most of the combinatorial structures defined in this section: "edge-colored directed graph," "vertex-colored directed graph," "ranked poset," "splitting poset". The letter " $P$ " is reserved for posets and "vertex-colored" posets that arise as posets of join irreducibles for distributive lattices. The letter " $L$ " is reserved for distributive lattices and "edge-colored" distributive lattices. All posets are finite. We identify a poset with its Hasse diagram.

Let $I$ be any set. An edge-colored directed graph with edges colored by the set $I$ is a directed graph $R$ with vertex set $\mathcal{V}(R)$ and directed-edge set $\mathcal{E}(R)$ together with a function edgecolor $_{R}$ : $\mathcal{E}(R) \longrightarrow I$ assigning to each edge of $R$ a color from the set $I$. If an edge $\mathbf{s} \rightarrow \mathbf{t}$ in $R$ is assigned $i \in I$, we write $\mathbf{s} \stackrel{i}{\rightarrow} \mathbf{t}$. See Figure 2.1. For $i \in I$, we let $\mathcal{E}_{i}(R)$ denote the set of edges in $R$ of color $i$, so $\mathcal{E}_{i}(R)=$ edgecolor $_{R}^{-1}(i)$. If $J$ is a subset of $I$, remove all edges from $R$ whose colors are not in $J$; connected components of the resulting edge-colored directed graph are called $J$-components of $R$. For any $\mathbf{t}$ in $R$ and any $J \subset I$, we let $\operatorname{comp}_{J}(\mathbf{t})$ denote the $J$-component of $R$ containing $\mathbf{t}$. The dual $R^{*}$ is the edge-colored directed graph whose vertex set $\mathcal{V}\left(R^{*}\right)$ is the set of symbols $\left\{\mathbf{t}^{*}\right\}_{\mathbf{t} \in R}$ together with colored edges $\mathcal{E}_{i}\left(R^{*}\right):=\left\{\mathbf{t}^{*} \stackrel{i}{\rightarrow} \mathbf{s}^{*} \mid \mathbf{s} \stackrel{i}{\rightarrow} \mathbf{t} \in \mathcal{E}_{i}(R)\right\}$ for each $i \in I$. Let $Q$ be another edge-colored directed graph with edge colors from $I$. If $R$ and $Q$ have disjoint vertex sets, then the disjoint sum $R \oplus Q$ is the expected edge-colored directed graph. If $\mathcal{V}(Q) \subseteq \mathcal{V}(R)$ and $\mathcal{E}_{i}(Q) \subseteq \mathcal{E}_{i}(R)$ for each $i \in I$, then $Q$ is an edge-colored subgraph of $R$. Let $R \times Q$ denote the expected edge-colored directed graph with vertex set $\mathcal{V}(R) \times \mathcal{V}(Q)$. The notion of isomorphism for edge-colored directed graphs is as expected. (See [ADLP] if any "expected" statement is unclear.) If $R$ is an edge-colored directed graph with edges colored by the set $I$, and if $\sigma: I \longrightarrow I^{\prime}$ is a mapping of sets, then we let $R^{\sigma}$ be the edge-colored directed graph with edge color function edgecolor $R^{\sigma}:=\sigma \circ$ edgecolor $_{R}$. We call $R^{\sigma}$ a recoloring of $R$. Observe that $\left(R^{*}\right)^{\sigma} \cong\left(R^{\sigma}\right)^{*}$. We similarly define a vertex-colored directed graph with a function vertexcolor $\operatorname{ve}_{R}: \mathcal{V}(R) \longrightarrow I$ that assigns colors to the vertices of $R$. In this context, we speak of the dual vertex-colored directed graph $R^{*}$, the disjoint sum of two vertex-colored directed graphs with disjoint vertex sets, isomorphism of vertex-colored directed graphs, recoloring, etc. See Figures 2.1 and 2.2.

For $\mathbf{s}$ and $\mathbf{t}$ in a poset $R$, there is a directed edge $\mathbf{s} \rightarrow \mathbf{t}$ in the Hasse diagram of $R$ if and only if $\mathbf{t}$ covers $\mathbf{s}$. So terminology that applies to directed graphs (connected, edge-colored, dual, vertex-colored, etc) will also apply to posets. The vertex $\mathbf{s}$ and the edge $\mathbf{s} \rightarrow \mathbf{t}$ are below $\mathbf{t}$, and the 
Figure 2.1: A vertex-colored poset $P$ and an edge-colored lattice $L$.
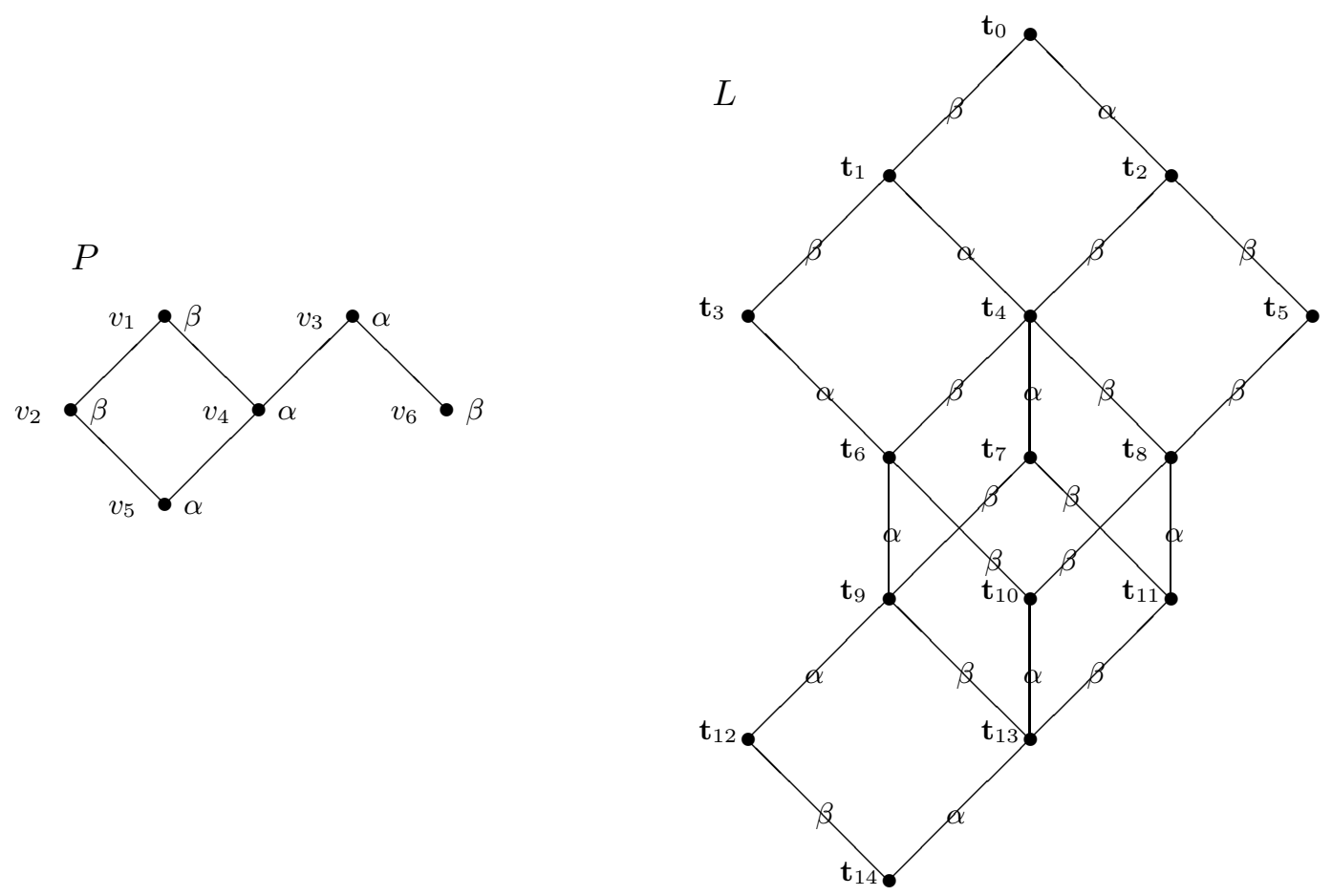

Figure 2.2: The disjoint sum of the $\beta$-components of the edge-colored lattice $L$ from Figure 2.1.
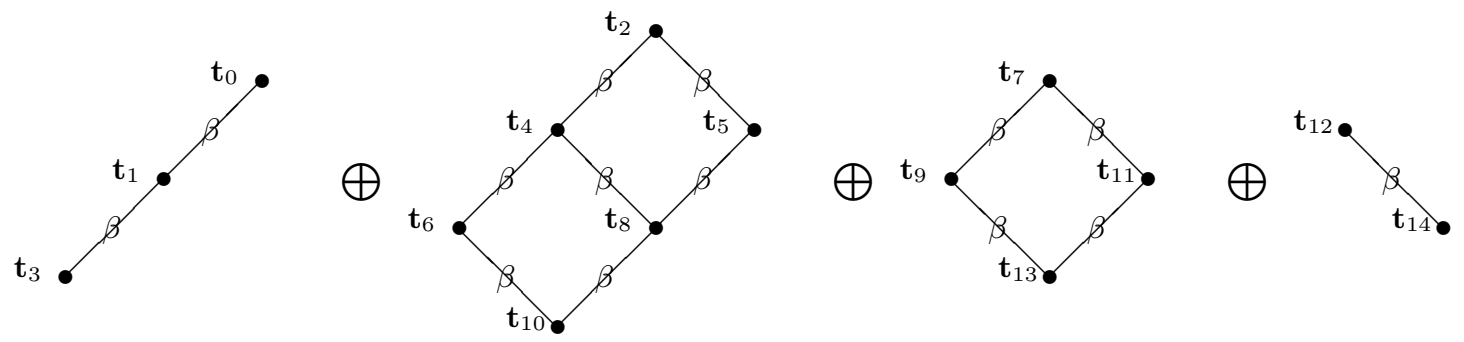

vertex $\mathbf{t}$ and the edge $\mathbf{s} \rightarrow \mathbf{t}$ are above $\mathbf{s}$. The vertex $\mathbf{s}$ is a descendant of $\mathbf{t}$, and $\mathbf{t}$ is an ancestor of $\mathbf{s}$. All edge-colored and vertex-colored directed graphs in this paper will turn out to be posets. For a directed graph $R$, a rank function is a surjective function $\rho: R \longrightarrow\{0, \ldots, l\}$ (where $l \geq 0$ ) with the property that if $\mathbf{s} \rightarrow \mathbf{t}$ in $R$, then $\rho(\mathbf{s})+1=\rho(\mathbf{t})$. If such a rank function exists then $R$ is the Hasse diagram for a poset - a ranked poset. We call $l$ the length of $R$ with respect to $\rho$, and the set $\rho^{-1}(i)$ is the $i$ th rank of $R$. In an edge-colored ranked poset $R, \operatorname{comp}_{i}(\mathbf{t})$ will be a ranked poset for each $\mathbf{t} \in R$ and $i \in I$. We let $l_{i}(\mathbf{t})$ denote the length of $\mathbf{c o m p}_{i}(\mathbf{t})$, and we let $\rho_{i}(\mathbf{t})$ denote the rank of $\mathbf{t}$ within this component. We define the depth of $\mathbf{t}$ in its $i$ component to be $\delta_{i}(\mathbf{t}):=l_{i}(\mathbf{t})-\rho_{i}(\mathbf{t})$. A ranked poset $R$ with rank function $\rho$ and length $l$ is rank symmetric if $\left|\rho^{-1}(i)\right|=\left|\rho^{-1}(l-i)\right|$ for $0 \leq i \leq l$. It is rank unimodal if there is an $m$ such that $\left|\rho^{-1}(0)\right| \leq\left|\rho^{-1}(1)\right| \leq \cdots \leq\left|\rho^{-1}(m)\right| \geq\left|\rho^{-1}(m+1)\right| \geq \cdots \geq\left|\rho^{-1}(l)\right|$.

The distributive lattice of order ideals of a poset $P$, partially ordered by subset containment, will be denoted $J(P)$. See [Sta2]. A coloring of the vertices of the poset $P$ gives a natural coloring of the edges of the distributive lattice $L=J(P)$, as follows: Given a function vertexcolor $P$ : 
$\mathcal{V}(P) \longrightarrow I$, we assign a covering relation $\mathbf{s} \rightarrow \mathbf{t}$ in $L$ the color $i$ and write $\mathbf{s} \stackrel{i}{\rightarrow} \mathbf{t}$ if $\mathbf{t} \backslash \mathbf{s}=\{u\}$ and vertexcolor $P(u)=i$. So $L$ becomes an edge-colored distributive lattice with edges colored by the set $I$; we write $L=J_{\text {color }}(P)$. The edge-colored lattice $L_{G_{2}}(0,1)$ of Figure 4.3 is obtained from the vertex-colored poset $P_{G_{2}}(0,1)$ of Figure 4.2 in this way. Note that $J_{\text {color }}\left(P^{*}\right) \cong\left(J_{\text {color }}(P)\right)^{*}$, $J_{\text {color }}\left(P^{\sigma}\right) \cong\left(J_{\text {color }}(P)\right)^{\sigma}$ (recoloring), and $J_{\text {color }}(P \oplus Q) \cong J_{\text {color }}(P) \times J_{\text {color }}(Q)$. An edge-colored poset $P$ has the diamond coloring property if whenever Hasse diagram for $P$, then $i=l$ and $j=k$. A necessary and sufficient condition for an edge-colored distributive lattice $L$ to be isomorphic (as an edge-colored poset) to $J_{\text {color }}(P)$ for some vertexcolored poset $P$ is for $L$ to have the diamond coloring property. Then for $\mathbf{s} \in L$ and $i \in I$, one can see that $\mathbf{c o m p}_{i}(\mathbf{s})$ is the Hasse diagram for a distributive lattice. In particular, $\mathbf{c o m p}_{i}(\mathbf{s})$ is a distributive sublattice of $L$ in the induced order, and a covering relation in $\mathbf{c o m p}_{i}(\mathbf{s})$ is also a covering relation in $L$.

Let $n \geq 1$. Let $\mathcal{D}$ be a Dynkin diagram with $n$ nodes which are indexed by the elements of a set $I$ such that $|I|=n$. The associated Cartan matrix is denoted $\left(\mathcal{D}_{i, j}\right)_{i, j \in I}$. Throughout this paper $\mathfrak{g}$ will denote the complex semisimple Lie algebra of rank $n$ with Chevalley generators $\left\{x_{i}, y_{i}, h_{i}\right\}_{i \in I}$ satisfying the Serre relations specified by the Cartan matrix for the Dynkin diagram at hand. Usually $I=\{1, \ldots, n\}$. In any Cartan matrix, $\mathcal{D}_{i, i}=2$ for $i \in I$. Figure 2.3 presents the off-diagonal entries $\mathcal{D}_{i, j}, i \neq j$, for the rank two semisimple Dynkin diagrams $A_{1} \oplus A_{1}, A_{2}, C_{2}$, and $G_{2}$. Two Dynkin diagrams $\mathcal{D}$ and $\mathcal{D}^{\prime}$ are isomorphic if under some one-to-one correspondence $\sigma: I \longrightarrow I^{\prime}$ we have $\mathcal{D}_{i, j}=\mathcal{D}_{\sigma(i), \sigma(j)}^{\prime}$ and $\mathcal{D}_{j, i}=\mathcal{D}_{\sigma(j), \sigma(i)}^{\prime}$. Let $E$ denote the Euclidean space equipped with an inner product $\langle\cdot, \cdot\rangle$ which contains the root system $\Phi$ associated to $\mathcal{D}$. The set of simple roots is denoted $\left\{\alpha_{i}\right\}_{i \in I}$. For a root $\alpha$, the coroot is $\alpha^{\vee}:=\frac{2 \alpha}{\langle\alpha, \alpha\rangle}$. The $(i, j)$-element $\mathcal{D}_{i, j}$ of the Cartan matrix is $\left\langle\alpha_{i}, \alpha_{j}^{\vee}\right\rangle$. The fundamental weights $\left\{\omega_{1}, \ldots, \omega_{n}\right\}$ form the basis for $E$ dual to the simple coroots $\left\{\alpha_{i}^{\vee}\right\}_{i=1}^{n}:\left\langle\omega_{j}, \alpha_{i}^{\vee}\right\rangle=\delta_{i, j}$. The lattice of weights $\Lambda$ is the set of all integral linear combinations of the fundamental weights. We coordinatize $\Lambda$ to obtain a one-to-one correspondence with $\mathbb{Z}^{n}$ as follows: identify $\omega_{i}$ with the axis vector $(0, \ldots, 1, \ldots, 0)$, where " 1 " is in the $i$ th position. For $i \in I, \alpha_{i}=\sum_{j \in I} \mathcal{D}_{i, j} \omega_{j}$. So the simple root $\alpha_{i}$ can be identified with the $i$ th row vector of the Cartan matrix. The Weyl group $W$ is generated by the simple reflections $s_{i}: E \rightarrow E$ for all $i \in I$ : Here $s_{i}(v)=v-\left\langle v, \alpha_{i}^{\vee}\right\rangle \alpha_{i}$ for $v \in E$.

Figure 2.3

\begin{tabular}{|c|c|c|c|c|c|}
\hline Subgraph & $\stackrel{l}{0}$ & ق & $\begin{array}{ll}i & j \\
\end{array}$ & $i<{ }^{j}$ & $\stackrel{i}{=}$ \\
\hline $\mathcal{D}_{i, j}, \quad \mathcal{D}_{j, i}$ & 0 & 0 & $-1, \quad-1$ & $-1,-2$ & $-1,-3$ \\
\hline
\end{tabular}

Vector spaces in this paper are complex and finite-dimensional. If $V$ is a $\mathfrak{g}$-module, then there is at least one basis $\mathcal{B}:=\left\{v_{\mathbf{s}}\right\}_{\mathbf{s} \in R}$ (where $R$ is an indexing set with $|R|=\operatorname{dim} V$ ) consisting of eigenvectors for the actions of the $h_{i}$ 's: for any $\mathbf{s}$ in $R$ and $i \in I$, there exists an integer $k_{i}(\mathbf{s})$ such that $h_{i} \cdot v_{\mathbf{s}}=k_{i}(\mathbf{s}) v_{\mathbf{s}}$. The weight of the basis vector $v_{\mathbf{s}}$ is the $\operatorname{sum} w t\left(v_{\mathbf{s}}\right):=\sum_{i \in I} k_{i}(\mathbf{s}) \omega_{i}$. We say $\mathcal{B}$ is a weight basis for $V$. If $\mu$ is a weight in $\Lambda$, then we let $V_{\mu}$ be the subspace of $V$ spanned by all basis vectors $v_{\mathbf{s}} \in \mathcal{B}$ such that $w t\left(v_{\mathbf{s}}\right)=\mu$. The subspace $V_{\mu}$ is independent of the choice of weight basis 
$\mathcal{B}$. The finite-dimensional irreducible $\mathfrak{g}$-modules are indexed by their "highest weights" $\lambda$ as these highest weights $\lambda$ run through the dominant weights $\Lambda^{+}$(the nonnegative linear combinations of the fundamental weights). The Lie algebra $\mathfrak{g}$ acts on the dual space $V^{*}$ by the rule $(z . f)(v)=-f(z \cdot v)$ for all $v \in V, f \in V^{*}$, and $z \in \mathfrak{g}$.

Let $R$ be a ranked poset whose Hasse diagram edges are colored with colors taken from $I,|I|=n$. For $i \in I$, find the connected components of the subgraph with edges $\mathcal{E}_{i}(R)$. For $i \in I$ and $\mathbf{s}$ in $R$, set $m_{i}(\mathbf{s}):=\rho_{i}(\mathbf{s})-\delta_{i}(\mathbf{s})=2 \rho_{i}(\mathbf{s})-l_{i}(\mathbf{s})$. Let $w t_{R}(\mathbf{s})$ be the $n$-tuple $\left(m_{i}(\mathbf{s})\right)_{i \in I}$. See Figure 4.4. Given a matrix $M=\left(M_{p, q}\right)_{p, q \in I}$, then for fixed $i \in I$ let $M^{(i)}$ be the $n$-tuple $\left(M_{i, j}\right)_{j \in I}$, the $i$ th row vector for $M$. We say $R$ satisfies the structure condition for $M$ if $w t_{R}(\mathbf{s})+M^{(i)}=w t_{R}(\mathbf{t})$ whenever $\mathbf{s} \stackrel{i}{\rightarrow} \mathbf{t}$ for some $i \in I$, that is, for all $j \in I$ we have $m_{j}(\mathbf{s})+M_{i, j}=m_{j}(\mathbf{t})$. Following [DLP], we say $R$ satisfies the $\mathfrak{g}$-structure condition if $M$ is the Cartan matrix for the Dynkin diagram $\mathcal{D}$ associated to $\mathfrak{g}$. In this case view $w t_{R}: R \longrightarrow \Lambda$ as the function given by $w t_{R}(\mathbf{s})=\sum_{j \in I} m_{j}(\mathbf{s}) \omega_{j}$. Then $R$ satisfies the $\mathfrak{g}$-structure condition if and only if for each simple root $\alpha_{i}$ we have $w t_{R}(\mathbf{s})+\alpha_{i}=w t_{R}(\mathbf{t})$ whenever $\mathbf{s} \stackrel{i}{\rightarrow} \mathbf{t}$ in $R$. (In [Don1] the edges of $R$ were said to "preserve weights".) This condition requires the color structure of $R$ to be compatible with the structure of the set of weights for a representation of $\mathfrak{g}$. The largest edge-colored distributive lattice of Figure 4.4 satisfies the structure condition for the $G_{2}$ Cartan matrix (Figure 4.1) and therefore satisfies the $G_{2}$-structure condition.

The following obvious lemma is used when the Dynkin diagram has symmetry or when other numberings of the Dynkin diagram are convenient.

Lemma 2.1 Let $\mathcal{D}$ and $\mathcal{D}^{\prime}$ be Dynkin diagrams with nodes indexed by $I$ and $I^{\prime}$ such that $\mathcal{D}$ and $\mathcal{D}^{\prime}$ are isomorphic under a one-to-one correspondence $\sigma: I \longrightarrow I^{\prime}$. Let $\mathfrak{g}$ and $\mathfrak{g}^{\prime}$ be the respective semisimple Lie algebras. Let $R$ be a ranked poset with edges colored by the set $I$, and consider the recoloring $R^{\sigma}$. Then $R$ satisfies the $\mathfrak{g}$-structure condition if and only if $R^{\sigma}$ satisfies the $\mathfrak{g}^{\prime}$-structure condition.

Let $w_{0}$ be the longest element of the Weyl group $W$ associated to $\mathfrak{g}$, as in Exercise 10.9 of [Hum]. When $w_{0}$ acts on $\Lambda$, then for each $i$ it sends $\alpha_{i} \mapsto-\alpha_{\sigma_{0}(i)}$ and $\omega_{i} \mapsto-\omega_{\sigma_{0}(i)}$, where $\sigma_{0}: I \longrightarrow I$ is some permutation of the node labels of the Dynkin diagram $\mathcal{D}$. Here $\sigma_{0}$ must be a symmetry of the Dynkin diagram, and since $w_{0}^{2}=i d$ in $W$ it is the case that $\sigma_{0}^{2}$ is the identity permutation. For any weight $\mu=\sum a_{i} \omega_{i}$ we have $-w_{0} \mu=\sum a_{i} \omega_{\sigma_{0}(i)}$. For connected Dynkin diagrams, $\sigma_{0}$ is trivial except in the cases $A_{n}(n \geq 2), D_{2 k+1}(k \geq 2)$, and $E_{6}$; in these cases it is the only nontrivial Dynkin diagram automorphism. Given an edge-colored poset $R$ with edges colored by the set $I$ of indices for the Dynkin diagram $\mathcal{D}$, we let $R^{\triangle}$ be the edge-colored poset $\left(R^{*}\right)^{\sigma_{0}}$ and call $R^{\triangle}$ the $\sigma_{0}$-recolored dual of $R$. Observe that $\left(R^{\triangle}\right)^{\triangle}=R$. We allow " $\triangle$ " to be applied to any vertex-colored poset $Q$ whose vertex colors correspond to nodes of a Dynkin diagram.

The group ring $\mathbb{Z}[\Lambda]$ has vector space basis $\left\{e_{\mu} \mid \mu \in \Lambda\right\}$ and multiplication rule $e_{\mu+\nu}=e_{\mu} e_{\nu}$. The Weyl group $W$ acts on $\mathbb{Z}[\Lambda]$ by the rule $\sigma . e_{\mu}:=e_{\sigma \mu}$. The character ring $\mathbb{Z}[\Lambda]^{W}$ for $\mathfrak{g}$ is the ring of $W$-invariant elements of $\mathbb{Z}[\Lambda]$; elements of $\mathbb{Z}[\Lambda]^{W}$ are characters for $\mathfrak{g}$. If $V$ is a representation of $\mathfrak{g}$, then the Weyl character for $V$ is $\chi(V):=\sum_{\mu \in \Lambda}\left(\operatorname{dim} V_{\mu}\right) e_{\mu} \in \mathbb{Z}[\Lambda]^{W}$. If $V$ is irreducible with highest weight $\lambda$, let $\chi_{\lambda}:=\chi(V)$. We call $\chi_{\lambda}$ an irreducible character. Let $A_{\mu}:=\sum_{\sigma \in W} \operatorname{det}(\sigma) e_{\sigma \mu}$. 
Let $\varrho:=\omega_{1}+\cdots+\omega_{n}$. It is well-known that $A_{\varrho}=e_{\varrho} \Pi\left(1-e_{-\alpha}\right)$, product taken over the positive roots $\alpha$. Weyl's character formula says that $\chi_{\lambda}$ is the unique element of $\mathbb{Z}[\Lambda]^{W}$ for which $A_{\varrho} \chi_{\lambda}=A_{\varrho+\lambda}$.

Let $V$ be a representation of $\mathfrak{g}$. A splitting system for $V$ (or for $\chi(V)$ ) is a pair $(\mathcal{T}$, weight), where $\mathcal{T}$ is a set and weight $: \mathcal{T} \longrightarrow \Lambda$ is a weight function such that $\chi(V):=\sum_{\mathbf{t} \in \mathcal{T}} e_{\text {weight }(\mathbf{t})}$. If $R$ is a ranked poset with edges colored by the set $\{1, \ldots, n\}$, if $R$ satisfies the structure condition for $\mathfrak{g}$, and if $\left(R, w t_{R}\right)$ is a splitting system for $V$, then we say $R$ is a splitting poset for $V$ (or for $\chi(V)$ ). This concept appears unnamed on p. 266 of [Don1] and as "labelling poset" in Corollary 5.3 of ADLP]. An edge-colored ranked poset $R$ for which $\left(R, w t_{R}\right)$ is a splitting system for an irreducible representation can fail to satisfy the structure condition for $\mathfrak{g}$. We use $z_{i}$ to denote $e_{\omega_{i}}$. If $R$ is a splitting poset for $V$, then $\chi(V)=\sum_{\mathbf{t} \in R}\left(z_{1}, \ldots, z_{n}\right)^{w t_{R}(\mathbf{t})}$, where $\left(z_{1}, \ldots, z_{n}\right)^{w t_{R}(\mathbf{t})}:=z_{1}^{m_{1}(\mathbf{t})} \ldots z_{n}^{m_{n}(\mathbf{t})}$. Here $\chi_{\lambda}$ is a Laurent polynomial in the indeterminates $z_{i}$ with nonnegative integer coefficients. We denote this polynomial by $\operatorname{char}_{\mathfrak{g}}\left(\lambda ; z_{1}, \ldots, z_{n}\right)$.

Lemma 2.2 Let $V$ be a representation for a semisimple Lie algebra $\mathfrak{g}$. Let $\mathfrak{g}^{\prime}$ be a semisimple Lie algebra isomorphic to $\mathfrak{g}$ obtained from an isomorphism $\sigma$ of Dynkin diagrams as in the statement of Lemma 2.1. Suppose $R$ is a splitting poset for $V$. Then the edge-colored poset $R^{*}$ is a splitting poset for the dual representation $V^{*}$ of $\mathfrak{g}, R^{\sigma}$ is a splitting poset for the $\mathfrak{g}^{\prime}$-module $V$, and $R^{\triangle}$ is a splitting poset for the $\mathfrak{g}$-module $V$.

Proof. The only assertion that does not immediately follow from the definitions and Lemma 2.1 is that $R^{\triangle}$ is a splitting poset for the $\mathfrak{g}$-module $V$. Write $V \cong V_{1} \oplus \cdots \oplus V_{k}$, a decomposition of $V$ into irreducible $\mathfrak{g}$-modules $V_{i}$ such that $V_{i}$ has highest weight $\mu_{i}$. The dual $\mathfrak{g}$-module $V^{*}$ has $R^{*}$ as a supporting graph; $V^{*}$ decomposes as $V_{1}^{*} \oplus \cdots \oplus V_{k}^{*}$, where each $V_{i}^{*}$ is irreducible with highest weight $-w_{0}\left(\mu_{i}\right)$ (cf. Exercise 21.6 of [Hum]). Recolor $R^{*}$ by applying the permutation $\sigma_{0}$ to obtain $R^{\triangle}$. Now view $V^{*}$ as a new $\mathfrak{g}$-module $U$ induced by the action $x_{i} \cdot v:=x_{\sigma_{0}(i)} \cdot v$ and $y_{i} \cdot v:=y_{\sigma_{0}(i)} \cdot v$ for each $i \in I$ and $v \in V^{*}$. It is apparent that $R^{\triangle}$ is a splitting poset for the $\mathfrak{g}$-module $U$. Let $U_{i}$ be the (irreducible) $\mathfrak{g}$-submodule of $U$ corresponding to $V_{i}^{*}$. One can see that the highest weight of $U_{i}$ is now $-w_{0}\left(-w_{0}\left(\mu_{i}\right)\right)$, which is just $\mu_{i}$. Hence $U$ is isomorphic to $V$.

Lemma 2.3 Let $V$ be an irreducible $\mathfrak{g}$-module. Then there is a connected splitting poset for $V$.

Proof. By Lemmas 3.1.A and 3.2.A of [Don1], any supporting graph for $V$ will do. By Lemma 3.1.F of [Don1], since $V$ is irreducible then any supporting graph for $V$ is necessarily connected. $\square$

This paragraph and Proposition 2.4 borrow from Sections 5 and 6 of [Pr1]. If we set

$$
x:=2 \sum_{i=1}^{n}\left[\sum_{j=1}^{n} \frac{2\left\langle\omega_{i}, \omega_{j}\right\rangle}{\left\langle\alpha_{j}, \alpha_{j}\right\rangle}\right] x_{i}, \quad y:=\sum y_{i}, \quad \text { and } \quad h:=2 \sum_{i=1}^{n}\left[\sum_{j=1}^{n} \frac{2\left\langle\omega_{i}, \omega_{j}\right\rangle}{\left\langle\alpha_{j}, \alpha_{j}\right\rangle}\right] h_{i},
$$

then $\mathfrak{s}:=\operatorname{span}\{x, y, h\}$ is a three-dimensional subalgebra of $\mathfrak{g}$ isomorphic to $\mathfrak{s l}(2, \mathbb{C})$. It is called a "principal three-dimensional subalgebra". Set $\varrho^{\vee}:=\sum_{i=1}^{n} \frac{2 \omega_{i}}{\left\langle\alpha_{i}, \alpha_{i}\right\rangle}$. Observe that $\left\langle\alpha_{i}, \varrho^{\vee}\right\rangle=1$ for $1 \leq i \leq n$. Let $V$ be a $\mathfrak{g}$-module. Let $R$ be a splitting poset for $V$. Then there exists a weight basis for $V$ which can be indexed by the elements of $R$, say $\left\{v_{\mathbf{t}}\right\}_{\mathbf{t} \in R}$, so that the weight of the basis vector $v_{\mathbf{t}}$ is $w t_{R}(\mathbf{t})$. One can check that $h . v_{\mathbf{t}}=2\left\langle w t_{R}(\mathbf{t}), \varrho^{\vee}\right\rangle v_{\mathbf{t}}$, so the set $\left\{2\left\langle w t_{R}(\mathbf{x}), \varrho^{\vee}\right\rangle\right\}_{\mathbf{x} \in R}$ 
consists of the integral weights for $V$ regarded as an $\mathfrak{s - m o d u l e . ~ C h o o s e ~ a n ~ e l e m e n t ~} \max$ in $R$ such that $2\left\langle w t_{R}(\mathbf{m a x}), \varrho^{\vee}\right\rangle$ is largest in the set $\left\{2\left\langle w t_{R}(\mathbf{x}), \varrho^{\vee}\right\rangle\right\}_{\mathbf{x} \in R}$, and choose min such that $2\left\langle w t_{R}(\mathbf{m i n}), \varrho^{\vee}\right\rangle$ is smallest. Symmetry of the integral weights for $V$ under the action of $\mathfrak{s} \cong \mathfrak{s l}(2, \mathbb{C})$ implies that $2\left\langle w t_{R}(\mathbf{m a x}), \varrho^{\vee}\right\rangle=-2\left\langle w t_{R}(\mathbf{m i n}), \varrho^{\vee}\right\rangle$. Set $l:=2\left\langle w t_{R}(\mathbf{m a x}), \varrho^{\vee}\right\rangle$. Since $R$ satisfies the $\mathfrak{g}$-structure condition, it follows that if $\mathbf{s} \stackrel{i}{\rightarrow} \mathbf{t}$ is an edge in $R$, then $w t_{R}(\mathbf{s})+\alpha_{i}=w t_{R}(\mathbf{t})$; therefore $\left\langle w t_{R}(\mathbf{s}), \varrho^{\vee}\right\rangle+1=\left\langle w t_{R}(\mathbf{t}), \varrho^{\vee}\right\rangle$. Suppose for the moment that $R$ is connected. Then the weights $\left\{2\left\langle w t_{R}(\mathbf{x}), \varrho^{\vee}\right\rangle\right\}_{\mathbf{x} \in R}$ all have the same parity. Consider the function $\rho: R \longrightarrow \mathbb{Z}$ given by $\rho(\mathbf{t}):=\frac{l}{2}+\left\langle w t_{R}(\mathbf{t}), \varrho^{\vee}\right\rangle$. Based on what we have seen so far, the range of $\rho$ is the set of integers $\{0, \ldots, l\}$, and hence $\rho$ is the rank function for $R$. Next consider the case that $V$ is irreducible with highest weight $\lambda$. Then $R$ need not be connected. However, since $V$ has a connected splitting poset by Lemma 2.3 , then the weights $\left\{2\left\langle w t_{R}(\mathbf{x}), \varrho^{\vee}\right\rangle\right\}_{\mathbf{x} \in R}$ all have the same parity. Thus the function $\rho: R \longrightarrow \mathbb{Z}$ given by $\rho(\mathbf{t}):=\frac{l}{2}+\left\langle w t_{R}(\mathbf{t}), \varrho^{\vee}\right\rangle$ will be a rank function for $R$ with range $\{0, \ldots, l\}$. Call $\rho$ the natural rank function for $R$. Since $V$ is irreducible, we can see that max is the unique element of $R$ with weight $w t_{R}(\mathbf{m a x})=\lambda$. Hence $l=2\left\langle\lambda, \varrho^{\vee}\right\rangle$. Next we define the rank generating function for $R$ to be $R G F_{\mathfrak{g}}(\lambda, q):=\sum_{i=0}^{l}\left|\rho^{-1}(i)\right| q^{i}=\sum_{\mathbf{t} \in R} q^{\rho(\mathbf{t})}$. This is the usual rank generating function for the ranked poset $R$. We do not refer to $R$ in the notation $R G F_{\mathfrak{g}}(\lambda, q)$ because: We have $\left|\rho^{-1}(i)\right|=\left|\left\{\mathbf{t} \in R \mid \frac{l}{2}+\left\langle w t_{R}(\mathbf{t}), \varrho^{\vee}\right\rangle=i\right\}\right|=\sum_{\mu} \operatorname{dim}\left(V_{\mu}\right)$, where the latter sum is over all weights $\mu$ such that $\frac{l}{2}+\left\langle\mu, \varrho^{\vee}\right\rangle=i$. Thus if $R^{\prime}$ is another naturally-ranked splitting poset for $V$, then corresponding ranks of $R$ and $R^{\prime}$ have the same size. To obtain the rank generating function identity in the following result we use the "principal specialization" of Weyl's character formula from Section 6 of [Pr1].

Proposition 2.4 Let $V$ be an irreducible $\mathfrak{g}$-module with highest weight $\lambda$, and let $R$ be a splitting poset for $V$ with the natural rank function identified in the preceding paragraph. (If $R$ is connected, then the natural rank function is the unique rank function.) Then $R$ is rank symmetric and rank unimodal, and

$$
R G F_{\mathfrak{g}}(\lambda, q)=\frac{\Pi_{\alpha \in \Phi^{+}}\left(1-q^{\langle\lambda+\varrho, \alpha\rangle}\right)}{\Pi_{\alpha \in \Phi^{+}}\left(1-q^{\langle\varrho, \alpha\rangle}\right)}
$$

Proof. Choose a connected splitting poset $R^{\prime}$ for $V$; the natural rank function for $R^{\prime}$ is the unique rank function. Then by Proposition 3.5 of [Don1], it follows that $R^{\prime}$ is rank symmetric and rank unimodal. From the observation of the next-to-last sentence of the paragraph preceding the proposition, we conclude that the naturally ranked poset $R$ is rank symmetric and rank unimodal. The principal specialization obtained from [Pr1] pp. 337-338 is for simple Lie algebras, but the same arguments are valid for semisimple Lie algebras. Apply this to obtain the rank generating function identity of the proposition statement.

\section{Grid posets and two-color grid posets}

Here we introduce general grid posets and two-color grid posets with purely combinatorial definitions. From Section 4 onward we will consider only the particular two-color grid posets called "g-semistandard" posets, whose structures are indexed by rank two Dynkin diagrams. Some (uncolored) grid posets are displayed in Figure 3.1; the poset $P$ in Figure 2.1 is a two-colored grid poset. 
In the general setting of this section, Lemma 3.1 and its related definitions provide for the decomposition of two-color grid posets into manageable pieces. Given $m \geq 1$, set $[m]:=\{1,2, \ldots, m\}$.

Given a finite poset $\left(P, \leq_{P}\right)$, a chain function for $P$ is a function chain : $P \longrightarrow[m]$ for some positive integer $m$ such that (1) chain $^{-1}(i)$ is a (possibly empty) chain in $P$ for $1 \leq i \leq m$, and (2) given any cover $u \rightarrow v$ in $P$, it is the case that either $\operatorname{chain}(u)=\operatorname{chain}(v)$ or $\operatorname{chain}(u)=\operatorname{chain}(v)+$ 1. A grid poset is a finite poset $\left(P, \leq_{P}\right)$ together with a chain function chain $: P \longrightarrow[m]$ for some $m \geq 1$. Depending on context, the notation $P$ can refer to the grid poset $\left(P, \leq_{P}\right.$, chain $\left.: P \longrightarrow[m]\right)$ or the underlying poset $\left(P, \leq_{P}\right)$. The conditions on chain imply that an element in a grid poset covers no more than two elements and is covered by no more than two elements.* Observe that if $i$ is the smallest (respectively largest) integer such that chain $^{-1}(i)$ is nonempty and if $u$ is the maximal (respectively minimal) element of $\operatorname{chain}^{-1}(i)$, then $u$ is a maximal (respectively minimal) element of the poset $P$. A grid poset $P$ is connected if and only if the Hasse diagram for the poset $P$ is connected. For $1 \leq i \leq m$ we set $\mathcal{C}_{i}:=\operatorname{chain}^{-1}(i)$. When we depict grid posets, the chains $\mathcal{C}_{i}$ will be directed from SW to NE. See Figure 3.1.

Figure 3.1: The six non-isomorphic connected grid posets with three elements.
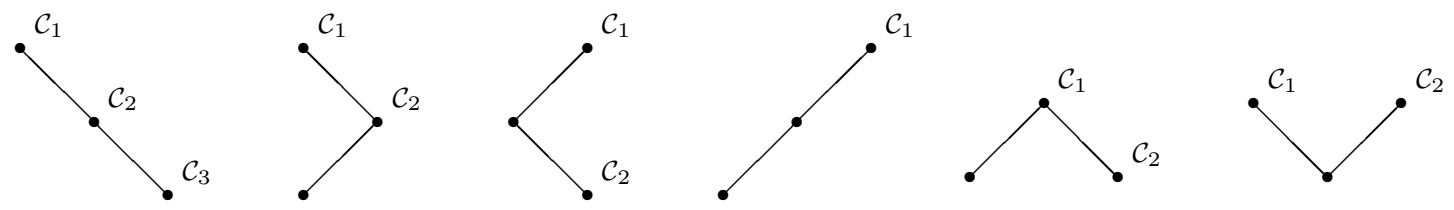

Let $\left(P, \leq_{P}\right.$, chain : $\left.P \longrightarrow[m]\right)$ be a grid poset. The dual grid poset $P^{*}$ is the dual poset $P^{*}$ together with the chain function chain* $: P^{*} \longrightarrow[m]$ given by chain* $\left(u^{*}\right)=m+1-\operatorname{chain}(u)$ for all $u \in P$. For $i=1,2$, let $P_{i}$ be a grid poset with chain function $\operatorname{chain}_{i}: P_{i} \longrightarrow\left[m_{i}\right]$ for some $m_{i} \geq 1$. A one-to-one correspondence $\phi: P_{1} \longrightarrow P_{2}$ is an isomorphism of grid posets if we have $u \rightarrow v$ in $P_{1}$

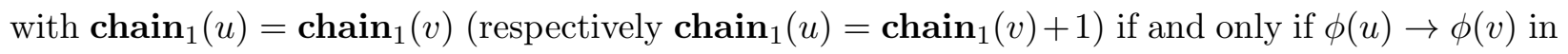

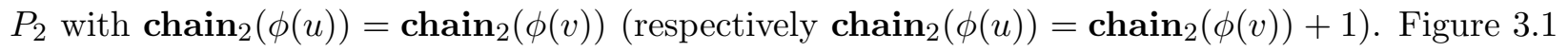
depicts each of the isomorphism classes of connected grid posets with three elements apiece. Given a nonempty grid poset $\left(P, \leq_{P}\right.$, chain $\left.: P \longrightarrow[m]\right)$, there exists some $m^{\prime} \geq 1$ and a surjective chain function chain $^{\prime}: P \longrightarrow\left[m^{\prime}\right]$ such that the grid poset $P$ is isomorphic to $\left(P, \leq_{P}\right.$, chain $\left.^{\prime}: P \longrightarrow\left[m^{\prime}\right]\right)$. If $P$ is connected, then this surjective chain function chain' is unique. We say $Q$ is a grid subposet of a given grid poset $P$ if (1) $Q$ is a subposet of $P$ in the induced order, and (2) whenever $u \rightarrow v$ is a covering relation in $Q$ then it is also a covering relation in $P$. In this case, we regard $Q$ with the chain function chain $\left.\right|_{Q}$ to be a grid poset on its own.

For a grid poset $\left(P, \leq_{P}\right.$, chain $\left.: P \longrightarrow[m]\right)$, let $\mathcal{T}_{P}$ be the totally ordered set whose elements are the elements of $P$ and whose ordering is given by the following rule: for distinct $u$ and $v$ in $P$ write $u<_{\mathcal{T}_{P}} v$ if and only if $(1) \operatorname{chain}(u)<\operatorname{chain}(v)$ or $(2) \operatorname{chain}(u)=\operatorname{chain}(v)$ with $v<_{P} u$.

${ }^{*}$ Motivation for terminology: For $m, n \geq 1$, let $\mathcal{G}$ be the directed graph with $\mathcal{V}(\mathcal{G})=\{(p, q) \in \mathbb{Z} \times \mathbb{Z} \mid 1 \leq p \leq n, 1 \leq$ $q \leq m\}$ and with $\mathcal{E}(\mathcal{G})=\{(p, q) \rightarrow(r, s)$ if $(r, s)-(p, q)=(1,0)$ or $(0,1)\}$. Refer to $\mathcal{G}$ as a "directed grid graph". Here $\mathcal{G}$ is the Hasse diagram for a poset obtained by rotating the plane counterclockwise through an angle of $45^{\circ}$,

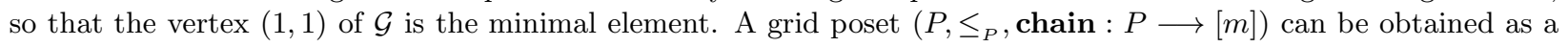
subgraph of a directed grid graph for an appropriately large $n$ by removing some vertices and some "NW" edges. 
Let $l:=|P|$. Number the vertices of $P v_{1}, v_{2}, \ldots, v_{l}$ so that $v_{p}<_{\mathcal{T}_{P}} v_{q}$ whenever $1 \leq p<q \leq l$. Let $L:=J(P)$ be the distributive lattice of order ideals of $P$. We simultaneously think of order ideals of $P$ as subsets of $P$ and as elements of $L$.

A two-color function for a grid poset $\left(P, \leq_{P}\right.$, chain $\left.: P \longrightarrow[m]\right)$ is a function color $: P \longrightarrow \Delta$ such that (1) $|\Delta|=2,(2) \operatorname{color}(u)=\operatorname{color}(v)$ if $\operatorname{chain}(u)=\operatorname{chain}(v)$, and (3) if $u$ and $v$ are in the same connected component of $P$ with $\operatorname{chain}(u)=\operatorname{chain}(v)+1$, then $\operatorname{color}(u) \neq \operatorname{color}(v)$. A two-color grid poset is a grid poset $\left(P, \leq_{P}\right.$, chain $\left.: P \longrightarrow[m]\right)$ together with a two-color function color $: P \longrightarrow \Delta$. In some contexts we will use the notation $P$ to refer to the two-color grid poset $\left(P, \leq_{P}\right.$, chain $: P \longrightarrow[m]$, color $\left.: P \longrightarrow \Delta\right)$. Two-color grid posets are vertex-colored posets. To a two-color grid poset $P$ we associate the edge-colored distributive lattice $L:=J_{\text {color }}(P)$, as in Section 2. The number of nonempty chains $\mathcal{C}_{i}$ in $P$ of color $\gamma \in \Delta$ gives an upper bound for the number of ancestors (respectively, descendants) an element in $L$ can have along edges of color $\gamma$. One can also see that any color $\gamma$ component of $L$ is poset-isomorphic to a product of chains. The dual two-color grid poset $P^{*}$ is the dual grid poset $P^{*}$ together with the two-color function color* $: P^{*} \longrightarrow \Delta$ given by $\operatorname{color}^{*}\left(u^{*}\right)=\operatorname{color}(u)$ for all $u \in P$. If $Q$ is a grid subposet of the two-color grid poset $P$, then $Q$ is a two-color grid poset with chain function chain $\left.\right|_{Q}$ and twocolor function color $\left.\right|_{Q}$. In this case we call $Q$ a two-color grid subposet of $P$. Two two-color grid posets $\left(P_{i}, \leq_{P_{i}}\right.$, chain $_{i}: P_{i} \longrightarrow\left[m_{i}\right]$, color $\left._{i}: P_{i} \longrightarrow \Delta\right)$ for $i=1,2$ are isomorphic if there is an isomorphism $\phi: P_{1} \longrightarrow P_{2}$ of grid posets such that $\operatorname{color}_{2}(\phi(u))=\operatorname{color}_{1}(u)$ for all $u$ in $P_{1}$. We will often take $\Delta:=\{\alpha, \beta\}$. When we switch (or reverse) the vertex colors of $P$ we replace the color function color $: P \longrightarrow\{\alpha, \beta\}$ with the color function color $^{\prime}: P \longrightarrow\{\alpha, \beta\}$ given by: $\operatorname{color}^{\prime}(v)=\alpha$ if $\operatorname{color}(v)=\beta$, and $\operatorname{color}^{\prime}(v)=\beta$ if $\operatorname{color}(v)=\alpha$. Similarly, one can switch (or reverse) the edge colors of $L$. In Figures 3.2 and 3.3 we depict eight two-color grid posets; the numbering of the vertices for each poset $P$ follows the total ordering $\mathcal{T}_{P}$. The vertex-colored poset $P$ of Figure 2.1 is a two-color grid poset. The lattice $L$ in that figure is $J_{\text {color }}(P)$.

In this paper the following definition is needed only for a comment in Section 4 and for preview statements of Theorems 6.1 and 6.2. (It is also needed in ADLP.) We say a two-color grid poset $P$ has the max property if $P$ is isomorphic to a two-color grid poset $\left(Q, \leq_{Q}\right.$, chain : $Q \longrightarrow[m]$, color : $Q \longrightarrow \Delta$ ) with a surjective chain function such that (1) if $u$ is any maximal element in the poset $Q$, then chain $(u) \leq 2$, and (2) if $v \neq u$ is another maximal element in $Q$, then $\operatorname{color}(u) \neq \operatorname{color}(v)$. Note that the dual two-color grid poset $P^{*}$ might fail to have the max property. The two-color grid posets of Figures 2.1, 3.2, and 3.3 have the max property.

Let $P$ be a grid poset with chain function chain $: P \longrightarrow[m]$. Suppose $P_{1}$ is a nonempty order ideal such that $P_{1} \neq P$. Regard $P_{1}$ and $P_{2}:=P \backslash P_{1}$ to be subposets of the poset $P$ in the induced order. Suppose that whenever $u$ is a maximal (respectively minimal) element of $P_{1}$ and $v$ is a maximal (respectively minimal) element of $P_{2}$, then $\operatorname{chain}(u) \leq \operatorname{chain}(v)$. Then we say that $P$ decomposes into $P_{1} \triangleleft P_{2}$, and we write $P=P_{1} \triangleleft P_{2}$. If no such order ideal $P_{1}$ exists, then we say the grid poset $P$ is indecomposable. See Figure 6.2. Note that if $P=P_{1} \triangleleft P_{2}$ and $u<v$ in $P$ with $u \in P_{2}$, then $v \in P_{2}$. Moreover, if $u \rightarrow v$ in $P$ with $u \in P_{1}$ and $v \in P_{2}$, then $\operatorname{chain}(u)=\operatorname{chain}(v)$. Also, if $u \rightarrow v$ is a covering relation in the poset $P_{i}$ for $i \in\{1,2\}$, note that $u \rightarrow v$ is also a 
covering relation in $P$. Hence each $P_{i}$ is a grid subposet of $P$. If $P$ is a grid poset that decomposes into $P_{1} \triangleleft Q$, and if $Q$ decomposes into $P_{2} \triangleleft P_{3}$, then $P=P_{1} \triangleleft\left(P_{2} \triangleleft P_{3}\right)$. But now observe that $P=\left(P_{1} \triangleleft P_{2}\right) \triangleleft P_{3}$. So we may write $P=P_{1} \triangleleft P_{2} \triangleleft P_{3}$. In general, if $P=P_{1} \triangleleft P_{2} \triangleleft \cdots \triangleleft P_{k}$, then each $P_{i}$ with chain function chain $\left.\right|_{P_{i}}$ is a grid subposet of $P$. Also, an order ideal $\mathbf{s}$ of $P$ may be expressed as the disjoint union $\left(\mathbf{s} \cap P_{1}\right) \cup\left(\mathbf{s} \cap P_{2}\right) \cup \cdots \cup\left(\mathbf{s} \cap P_{k}\right)$, where each $\mathbf{s} \cap P_{i}$ is an order ideal in $P_{i}$. If in addition $P=P_{1} \triangleleft P_{2} \triangleleft \cdots \triangleleft P_{k}$ is a two-color grid poset with two-color function color, then each $P_{i}$ with chain function chain $\left.\right|_{P_{i}}$ and two-color function color $\left.\right|_{P_{i}}$ is a two-color grid subposet of $P$. Here $P_{1} \triangleleft P_{2} \triangleleft \cdots \triangleleft P_{k}$ is a decomposition of $P$ into two-color grid posets.

Consider a two-color grid poset $\left(P, \leq_{P}\right.$, chain $: P \longrightarrow[m]$, color $\left.: P \longrightarrow\{\alpha, \beta\}\right)$ with edgecolored distributive lattice $L=J_{\text {color }}(P)$. For each $\mathbf{s}$ in $L$ we can view the quantity $w t_{L}(\mathbf{s})$ as the pair $\left(2 \rho_{\alpha}(\mathbf{s})-l_{\alpha}(\mathbf{s}), 2 \rho_{\beta}(\mathbf{s})-l_{\beta}(\mathbf{s})\right)$ in $\mathbb{Z} \times \mathbb{Z}$. The mapping $w t_{L}: L \longrightarrow \mathbb{Z} \times \mathbb{Z}$ is the lattice weight function for $L$. If $P=P_{1} \triangleleft P_{2} \triangleleft \cdots \triangleleft P_{k}$, then for each $i$ we let $L_{i}:=J_{\text {color }}\left(P_{i}\right)$ be the edge-colored lattice for the two-color grid subposet $P_{i}$ of $P$. Then $w t_{L_{i}}$ denotes the lattice weight function for $L_{i}$. We let $\rho_{\alpha}^{(i)}$ and $l_{\alpha}^{(i)}$ (respectively $\rho_{\beta}^{(i)}$ and $l_{\beta}^{(i)}$ ) denote the rank and length functions for color $\alpha$ (respectively color $\beta$ ) for $L_{i}$.

Lemma 3.1 Let $\left(P, \leq_{P}\right.$, chain $: P \longrightarrow[m]$, color $\left.: P \longrightarrow\{\alpha, \beta\}\right)$ be a two-color grid poset, and suppose $P$ decomposes into $P=P_{1} \triangleleft P_{2} \triangleleft \cdots \triangleleft P_{k}$. Keep the notation of the preceding paragraph. (1) Let $\gamma \in \Delta=\{\alpha, \beta\}$, and let $\mathbf{s}$ be an element of $L=J_{\text {color }}(P)$. Then

$$
\rho_{\gamma}(\mathbf{s})=\sum_{i=1}^{k} \rho_{\gamma}^{(i)}\left(\mathbf{s} \cap P_{i}\right), \quad l_{\gamma}(\mathbf{s})=\sum_{i=1}^{k} l_{\gamma}^{(i)}\left(\mathbf{s} \cap P_{i}\right), \quad \text { and } \quad w t_{L}(\mathbf{s})=\sum_{i=1}^{k} w t_{L_{i}}\left(\mathbf{s} \cap P_{i}\right) .
$$

(2) Consequently, if there is a $2 \times 2$ matrix $M=\left(M_{\iota, \kappa}\right)_{(\iota, \kappa) \in \Delta \times \Delta}$ such that each edge-colored distributive lattice $L_{i}=J_{\text {color }}\left(P_{i}\right)$ satisfies the structure condition for $M$, then $L$ satisfies the structure condition for $M$ as well.

Proof. First we show how (2) follows from (1). Given an edge $\mathbf{s} \stackrel{\gamma}{\rightarrow} \mathbf{t}$ in $L$, then it is the case that for some $j$ with $1 \leq j \leq k$ we have $\mathbf{s} \cap P_{j} \stackrel{\gamma}{\rightarrow} \mathbf{t} \cap P_{j}$ in $L_{j}$ while for $1 \leq i \leq k$ with $i \neq j$ we have $\mathbf{s} \cap P_{i}=\mathbf{t} \cap P_{i}$. Since $L_{j}$ satisfies the structure condition, we see that $w t_{L_{j}}\left(\mathbf{s} \cap P_{j}\right)+M^{(\gamma)}=w t_{L_{j}}\left(\mathbf{t} \cap P_{j}\right)$. For $i \neq j$ we have $w t_{L_{i}}\left(\mathbf{s} \cap P_{i}\right)=w t_{L_{i}}\left(\mathbf{t} \cap P_{i}\right)$. By (1) it follows that $w t_{L}(\mathbf{s})+M^{(\gamma)}=w t_{L}(\mathbf{t})$.

The results in (1) for general $k$ follow by induction once we prove the results for $k=2$. So let $k=2, \mathbf{s} \in L$, and $\gamma \in\{\alpha, \beta\}$. It suffices to show that $\rho_{\gamma}(\mathbf{s})=\rho_{\gamma}^{(1)}\left(\mathbf{s} \cap P_{1}\right)+\rho_{\gamma}^{(2)}\left(\mathbf{s} \cap P_{2}\right)$ and $l_{\gamma}(\mathbf{s})=l_{\gamma}^{(1)}\left(\mathbf{s} \cap P_{1}\right)+l_{\gamma}^{(2)}\left(\mathbf{s} \cap P_{2}\right)$. Let $\mathbf{r}_{0}, \mathbf{r}_{1}, \ldots$ be the sequence with $\mathbf{r}_{0}:=\mathbf{s}$ and $\mathbf{r}_{j+1}:=\mathbf{r}_{j} \backslash\left\{v_{i_{j+1}}\right\}$, where $j \geq 0$ and $v_{i_{j+1}}$ is the smallest vertex in $\mathcal{T}_{P}$ of color $\gamma$ that can be removed from $\mathbf{r}_{j}$ so that $\mathbf{r}_{j+1}$ is an order ideal of $P$. Let $\mathbf{r}_{q}$ be the terminal element of the sequence. Observe that $i_{1}<i_{2}<\cdots<i_{q}$. We have $\mathbf{r}_{q} \stackrel{\gamma}{\rightarrow} \mathbf{r}_{q-1} \stackrel{\gamma}{\rightarrow} \cdots \stackrel{\gamma}{\rightarrow} \mathbf{r}_{1} \stackrel{\gamma}{\rightarrow} \mathbf{r}_{0}=\mathbf{s}$. Similarly define a sequence $\mathbf{u}_{0}, \mathbf{u}_{1}, \ldots$ where $\mathbf{u}_{0}:=\mathbf{s}$ and $\mathbf{u}_{s+1}:=\mathbf{u}_{s} \cup\left\{v_{r_{s+1}}\right\}$ where $s \geq 0$ and $v_{r_{s+1}}$ is the largest element in $\mathcal{T}_{P}$ of color $\gamma$ not in $\mathbf{u}_{s}$ that can be added to $\mathbf{u}_{s}$ so that $\mathbf{u}_{s+1}$ is an order ideal of $P$. Let $\mathbf{u}_{p}$ be the terminal element of the sequence. Observe that $r_{1}>r_{2}>\cdots>r_{p}$. We have $\mathbf{s}=\mathbf{u}_{0} \stackrel{\gamma}{\rightarrow} \cdots \stackrel{\gamma}{\rightarrow} \mathbf{u}_{p-1} \stackrel{\gamma}{\rightarrow} \mathbf{u}_{p}$. Since $\operatorname{comp}_{\gamma}(\mathbf{s})$ is the Hasse diagram for a distributive lattice, and since $\mathbf{r}_{q}$ and $\mathbf{u}_{p}$ are respectively 
Figure 3.2: Depicted below are four two-color grid posets each possessing the max property. (Each is the $\mathfrak{g}$-semistandard poset $P_{\mathfrak{g}}^{\beta \alpha}(2,2)$ of $\S 4$ for the indicated rank two semisimple Lie algebra $\mathfrak{g}$.)

$$
\mathfrak{g}=A_{1} \oplus A_{1}
$$
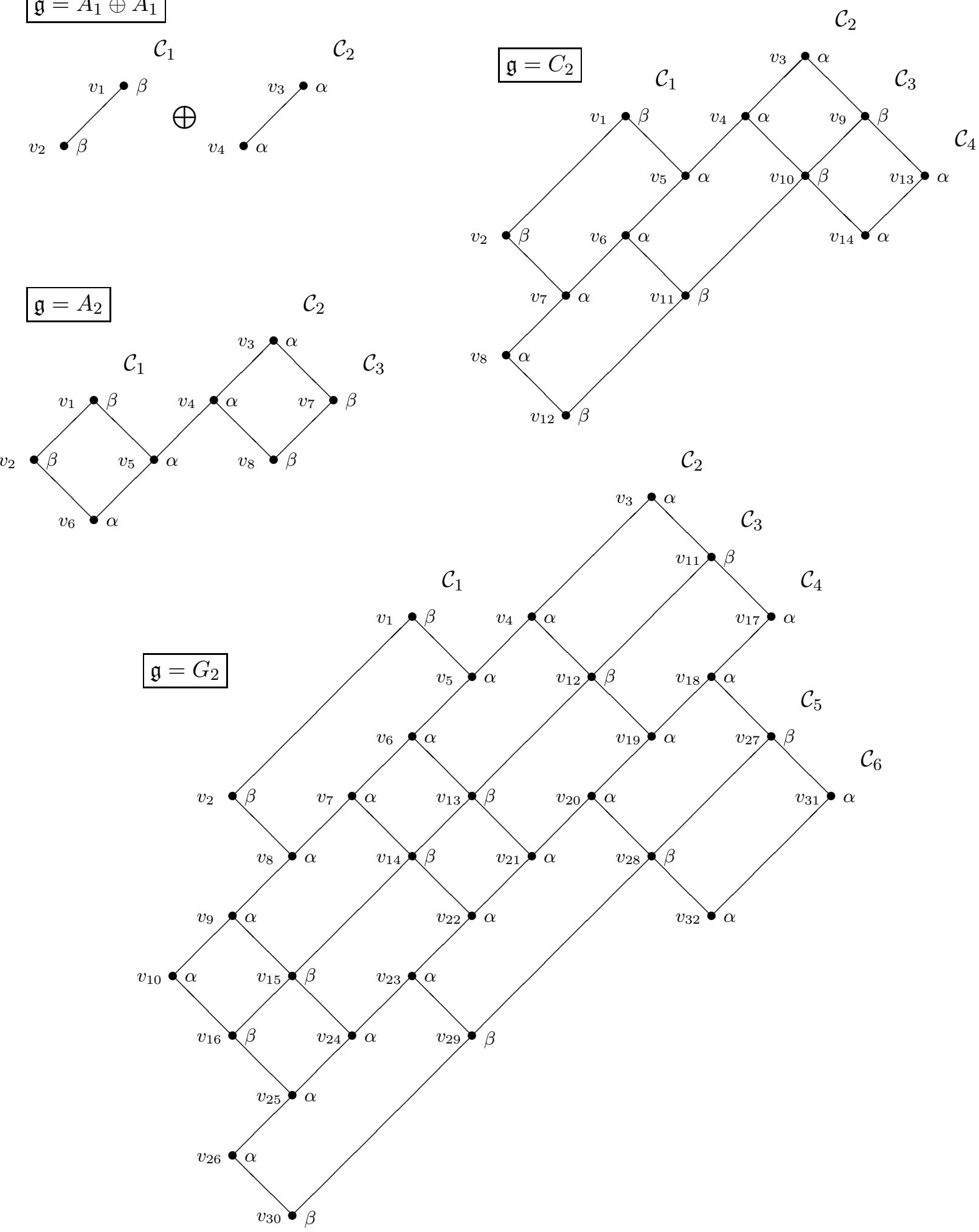
Figure 3.3: Depicted below are four two-color grid posets each possessing the max property. (Each is the $\mathfrak{g}$-semistandard poset $P_{\mathfrak{g}}^{\alpha \beta}(2,2)$ of $\S 4$ for the indicated rank two semisimple Lie algebra $\mathfrak{g}$.)

$$
\mathfrak{g}=A_{1} \oplus A_{1}
$$
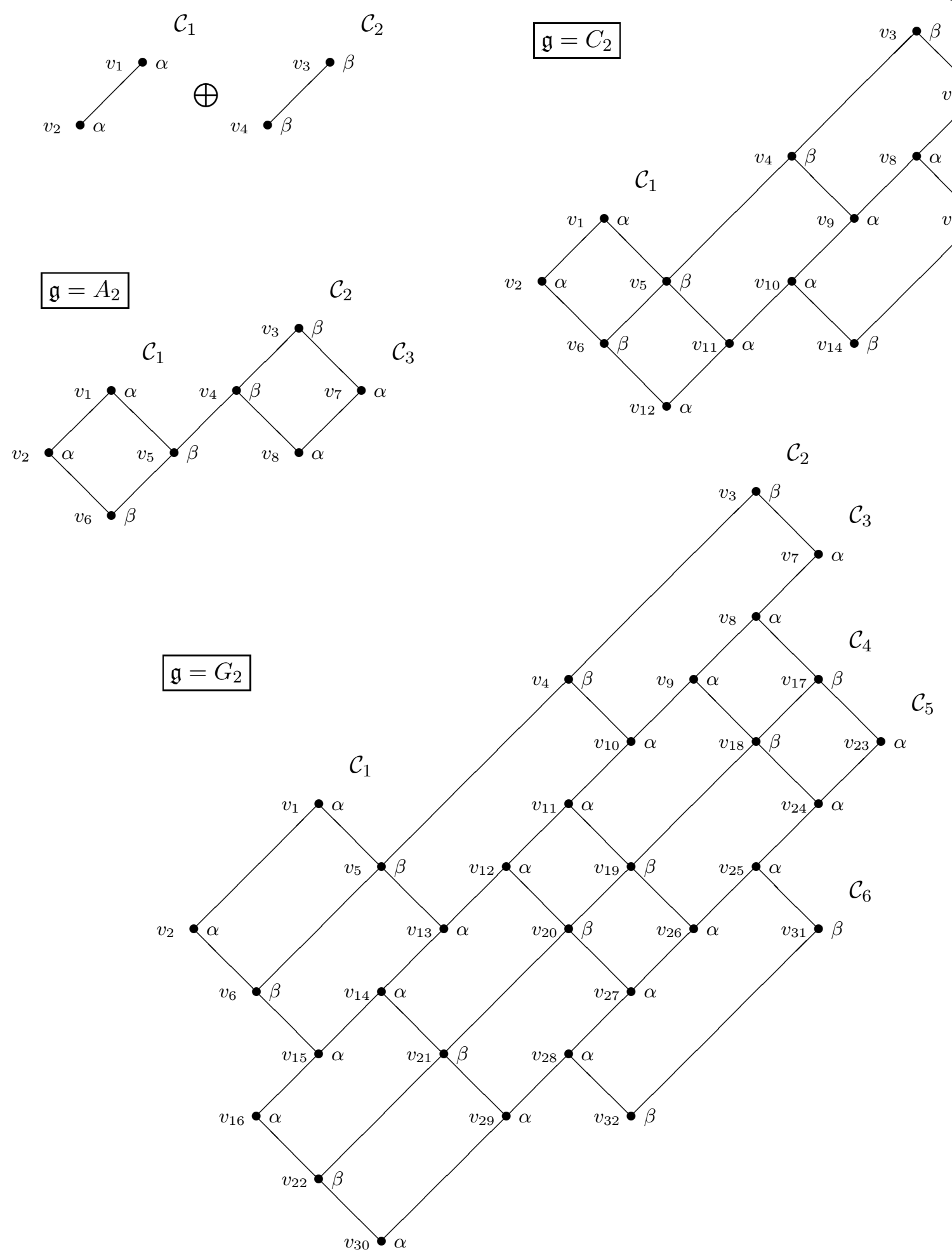
a minimal and a maximal element in $\operatorname{comp}_{\gamma}(\mathbf{s})$, then it follows that $\mathbf{r}_{q}$ and $\mathbf{u}_{p}$ are respectively the unique minimal and the unique maximal element of $\mathbf{c o m p}_{\gamma}(\mathbf{s})$. Then $\rho_{\gamma}(\mathbf{s})=q$ and $l_{\gamma}(\mathbf{s})=p+q$.

Reorganize the sequence $\left(v_{i_{1}}, \ldots, v_{i_{q}}\right)$ as follows: write $\left(v_{k_{1}}, \ldots, v_{k_{q^{\prime}}}, v_{k_{q^{\prime}+1}}, \ldots, v_{k_{q}}\right)$, where the vertices $v_{k_{1}}, \ldots, v_{k_{q^{\prime}}}$ are all in $P_{2}$ with $k_{1}<\cdots<k_{q^{\prime}}$, and the vertices $v_{k_{q^{\prime}+1}}, \ldots, v_{k_{q}}$ are all in $P_{1}$ with $k_{q^{\prime}+1}<\cdots<k_{q}$. Set $\mathbf{r}_{0}^{\prime}:=\mathbf{s}$ and for $j \geq 0$ set $\mathbf{r}_{j+1}^{\prime}:=\mathbf{r}_{j}^{\prime} \backslash\left\{v_{k_{j+1}}\right\}$. We claim that each $\mathbf{r}_{j+1}^{\prime}$ is an order ideal of $P$, and if $0 \leq j<q^{\prime}$ (respectively $q^{\prime} \leq j<q$ ) then $v_{k_{j+1}}$ is the smallest element in $\mathcal{T}_{P}$ of color $\gamma$ that is also in $P_{2}$ (respectively $P_{1}$ ) that can be removed from $\mathbf{r}_{j}^{\prime}$ so that $\mathbf{r}_{j+1}^{\prime}$ is an order ideal of $P$. (If so, we have a path $\mathbf{r}_{q}=\mathbf{r}_{q}^{\prime} \stackrel{\gamma}{\rightarrow} \mathbf{r}_{q-1}^{\prime} \stackrel{\gamma}{\rightarrow} \cdots \stackrel{\gamma}{\rightarrow} \mathbf{r}_{1}^{\prime} \stackrel{\gamma}{\rightarrow} \mathbf{r}_{0}^{\prime}=\mathbf{r}_{0}=\mathbf{s}$ in L.) Proceed by induction on $j$. The statement follows if we can show that $v_{k_{j+1}}$ is maximal in $\mathbf{r}_{j}^{\prime}$. First suppose $0 \leq j<q^{\prime}$. If $v_{k_{j+1}}$ is not maximal in $\mathbf{r}_{j}^{\prime}$, then $v_{k_{j+1}}<v$ for some other maximal element $v$ in $\mathbf{r}_{j}^{\prime}$. It must be the case that $v$ is one of $\left(v_{k_{j+2}}, \ldots, v_{k_{q^{\prime}}}, v_{k_{q^{\prime}+1}}, \ldots, v_{k_{q}}\right)$; otherwise one could not descend from $\mathbf{r}_{j}^{\prime}$ to $\mathbf{r}_{q}$ in $L=J_{\text {color }}(P)$ along edges corresponding to vertices from $\left(v_{k_{j+2}}, \ldots, v_{k_{q^{\prime}}}, v_{k_{q^{\prime}+1}}, \ldots, v_{k_{q}}\right)$. It cannot be the case that $v$ is one of $\left(v_{k_{j+2}}, \ldots, v_{k_{q^{\prime}}}\right)$; otherwise $k_{1}<\cdots<k_{j+1}<k_{j+2}<\cdots<k_{q^{\prime}}$ implies that $v$ is larger than $v_{k_{j+1}}$ in the total order $\mathcal{T}_{P}$, violating the fact that $v_{k_{j+1}}<v$ in $P$. And it cannot be the case that $v$ is one of $\left(v_{k_{q^{\prime}+1}}, \ldots, v_{k_{q}}\right)$ since these are elements of $P_{1}$ and $v_{k_{j+1}}$ is in $P_{2}$. So for $0 \leq j<q^{\prime}$, the vertex $v_{k_{j+1}}$ is maximal in $\mathbf{r}_{j}^{\prime}$. Second, suppose that $q^{\prime} \leq j<q$. If $v_{k_{j+1}}$ is not maximal in $\mathbf{r}_{j}^{\prime}$, then by reasoning similar to the preceding case we have $v_{k_{j+1}}<v$ for some element $v$ from $\left(v_{k_{j+2}}, \ldots, v_{k_{q}}\right)$. But this violates the fact that $v_{k_{j+1}}$ precedes $v$ in the total order $\mathcal{T}_{P}$ since $k_{q^{\prime}}<\cdots<k_{j+1}<k_{j+2}<\cdots<k_{q}$. So for $q^{\prime} \leq j<q$, the vertex $v_{k_{j+1}}$ is maximal in $\mathbf{r}_{j}^{\prime}$. This concludes our induction on $j$.

Let $\mathbf{r}^{(1)}$ be the unique minimal element in the $\gamma$-component $\operatorname{comp}_{\gamma}^{(1)}\left(\mathbf{s} \cap P_{1}\right)$ of $\mathbf{s} \cap P_{1}$ in the edge-colored distributive lattice $L_{1}=J_{\text {color }}\left(P_{1}\right)$. We claim that $\mathbf{r}^{(1)}=\mathbf{x}$, where $\mathbf{x}:=\left(\mathbf{s} \cap P_{1}\right) \backslash$ $\left\{v_{k_{q^{\prime}+1}}, \ldots, v_{k_{q}}\right\}$. Now $\mathbf{x}$ is an order ideal of $P_{1}$ since $\mathbf{x}=\mathbf{r}_{q}^{\prime} \cap P_{1}=\mathbf{r}_{q} \cap P_{1}$. Also, $\mathbf{x} \in \mathbf{c o m p} \mathbf{p}_{\gamma}^{(1)}\left(\mathbf{s} \cap P_{1}\right)$ since $\mathbf{s} \cap P_{1}=\mathbf{r}_{q^{\prime}}^{\prime} \cap P_{1}$ and the path $\mathbf{x} \stackrel{\gamma}{\rightarrow}\left(\mathbf{r}_{q-1}^{\prime} \cap P_{1}\right) \stackrel{\gamma}{\rightarrow} \cdots \stackrel{\gamma}{\rightarrow}\left(\mathbf{r}_{q^{\prime}+1}^{\prime} \cap P_{1}\right) \stackrel{\gamma}{\rightarrow}\left(\mathbf{r}_{q^{\prime}}^{\prime} \cap P_{1}\right)$ stays in $\operatorname{comp}_{\gamma}^{(1)}\left(\mathbf{s} \cap P_{1}\right)$. If $\mathbf{r}^{(1)} \neq \mathbf{x}$, then $\mathbf{r}^{(1)}<\mathbf{x}$. In this case let $u \in \mathbf{x}$ be any color $\gamma$ vertex such that $\mathbf{x} \backslash\{u\}$ is an order ideal of $P_{1}$. Let $\mathcal{C}_{i}$ be the chain in $P$ that contains $u$. Note that $u$ is not maximal in $\mathbf{r}_{q} \subseteq P$, and hence $u \rightarrow u^{\prime}$ is a covering relation in $P$ for some $u^{\prime}$ in $\mathbf{r}_{q}$. We refer to the following as observation (*): If $w$ is any element of $P$ such that $u \rightarrow w$ and $w \in \mathbf{r}_{q}$, then $w \in P_{2}$. (Otherwise $w \in P_{1}$, so that $w \in \mathbf{x}$, and then $\mathbf{x} \backslash\{u\}$ cannot be an order ideal of $P_{1}$.) In particular, $u^{\prime} \in P_{2}$. We claim that $u^{\prime} \notin\left\{v_{k_{1}}, \ldots, v_{k_{q^{\prime}}}\right\}$. Indeed, if $u^{\prime} \in\left\{v_{k_{1}}, \ldots, v_{k_{q^{\prime}}}\right\}$, then since $u \notin\left\{v_{k_{q^{\prime}+1}}, \ldots, v_{k_{q}}\right\}$, it must be the case that $u \rightarrow u^{\prime \prime}$ for some $u^{\prime \prime} \in \mathbf{r}_{q}$ in $\mathcal{C}_{i-1}$. By observation (*), the element $u^{\prime \prime}$ is in $P_{2}$. But a covering relation in $P$ between elements of $P_{1}$ and elements of $P_{2}$ can only occur along the chains $\mathcal{C}_{1}, \ldots, \mathcal{C}_{m}$. Therefore $u^{\prime \prime} \in \mathcal{C}_{i}$, which contradicts the fact that $u^{\prime \prime} \in \mathcal{C}_{i-1}$. So it must be the case that $u^{\prime} \notin\left\{v_{k_{1}}, \ldots, v_{k_{q^{\prime}}}\right\}$. It follows that $u^{\prime}<u^{\prime \prime}$ for some $u^{\prime \prime} \in \mathbf{r}_{q}$ in $\mathcal{C}_{i-1}$. Let $v$ be a maximal element in $P$ such that $u^{\prime \prime} \leq v$. Note that $v \in P_{2}$ since $u^{\prime \prime} \in P_{2}$. Moreover, $v \in \mathcal{C}_{j}$ with $j \leq i-1$. Next suppose $u \rightarrow z$ for some $z \in P_{1}$. Since $z \in P_{1}$, then $z \neq u^{\prime}$. Therefore $z \in \mathcal{C}_{i-1}$. Therefore $z \leq u^{\prime \prime}$. But since $u^{\prime \prime}$ is in the order ideal $\mathbf{r}_{q}$, it follows that $z \in \mathbf{r}_{q}$. But by observation (*), it now follows that $z \in P_{2}$. This contradicts our hypothesis that $z \in P_{1}$. In particular, $u$ must be a maximal element in $P_{1}$. So $u \in \mathcal{C}_{i}$ is a maximal element in $P_{1}$ and $v \in \mathcal{C}_{j}$ is a maximal element 
in $P_{2}$, and $j<i$. This violates the fact that $P$ decomposes into $P_{1} \triangleleft P_{2}$. So $\mathbf{r}^{(1)}=\mathbf{x}$, and hence $\rho_{\gamma}^{(1)}\left(\mathbf{s} \cap P_{1}\right)=q-q^{\prime}$.

Let $\mathbf{r}^{(2)}$ be the unique minimal element in the $\gamma$-component $\operatorname{comp}_{\gamma}^{(2)}\left(\mathbf{s} \cap P_{2}\right)$ of $\mathbf{s} \cap P_{2}$ in the edge-colored distributive lattice $L_{2}=J_{\text {color }}\left(P_{2}\right)$. We claim that $\mathbf{r}^{(2)}=\mathbf{y}$, where $\mathbf{y}:=\left(\mathbf{s} \cap P_{2}\right) \backslash$ $\left\{v_{k_{1}}, \ldots, v_{k_{q^{\prime}}}\right\}$. Now $\mathbf{y}$ is an order ideal of $P_{2}$ since $\mathbf{y}=\mathbf{r}_{q^{\prime}}^{\prime} \cap P_{2}=\mathbf{r}_{q} \cap P_{2}$. Also, $\mathbf{y} \in \mathbf{c o m p}_{\gamma}^{(2)}\left(\mathbf{s} \cap P_{2}\right)$ since the path $\mathbf{y} \stackrel{\gamma}{\rightarrow}\left(\mathbf{r}_{q^{\prime}-1}^{\prime} \cap P_{2}\right) \stackrel{\gamma}{\rightarrow} \cdots \stackrel{\gamma}{\rightarrow}\left(\mathbf{r}_{1}^{\prime} \cap P_{2}\right) \stackrel{\gamma}{\rightarrow}\left(\mathbf{r}_{0}^{\prime} \cap P_{2}\right)$ stays in $\mathbf{c o m p}_{\gamma}^{(2)}\left(\mathbf{s} \cap P_{2}\right)$. If $\mathbf{r}^{(2)} \neq \mathbf{y}$, then $\mathbf{r}^{(2)}<\mathbf{y}$. In this case let $u \in \mathbf{y}$ be any color $\gamma$ vertex such that $\mathbf{y} \backslash\{u\}$ is an order ideal of $P_{2}$. In particular, $u$ is a maximal element in $\mathbf{y}$. Let $w$ be any element of $\mathbf{r}_{q}$ with $u \neq w$. If $w \in P_{2}$, then $w \in \mathbf{y}$, so $u \nless w$. If $w \in P_{1}$, then by properties of the decomposition of $P$ into $P_{1} \triangleleft P_{2}$, it cannot be the case that $u<w$. Therefore $u$ is a maximal element of $\mathbf{r}_{q}$ of color $\gamma$. But this contradicts the fact that $\mathbf{r}_{q}$ is the minimal element in $\operatorname{comp}_{\gamma}(\mathbf{s})$. So it is not the case that $\mathbf{r}^{(2)}<\mathbf{y}$. Therefore $\mathbf{r}^{(2)}=\mathbf{y}$, and so $\rho_{\gamma}^{(2)}\left(\mathbf{s} \cap P_{2}\right)=q^{\prime}$. Combine this with $\rho_{\gamma}^{(1)}\left(\mathbf{s} \cap P_{1}\right)=q-q^{\prime}$ to see that $\rho_{\gamma}(\mathbf{s})=q=\left(q-q^{\prime}\right)+q^{\prime}=\rho_{\gamma}^{(1)}\left(\mathbf{s} \cap P_{1}\right)+\rho_{\gamma}^{(2)}\left(\mathbf{s} \cap P_{2}\right)$.

The dual $P^{*}$ may be viewed as a two-color grid poset that decomposes into $P_{2}^{*} \triangleleft P_{1}^{*}$. Order ideals of $P^{*}$ are complements of order ideals of $P$. Then arguments analogous to those above apply to the complements of elements of the sequence $\mathbf{s}=\mathbf{u}_{0}, \mathbf{u}_{1}, \ldots, \mathbf{u}_{p}$. So we obtain: $\rho_{\gamma}^{*}(P \backslash \mathbf{s})=\rho_{\gamma}^{*(2)}((P \backslash \mathbf{s}) \cap$ $\left.P_{2}\right)+\rho_{\gamma}^{*(1)}\left((P \backslash \mathbf{s}) \cap P_{1}\right)$. Note that $(P \backslash \mathbf{s}) \cap P_{i}=P_{i} \backslash\left(\mathbf{s} \cap P_{i}\right)$ for $i \in\{1,2\}$. Now $l_{\gamma}(\mathbf{s})=\rho_{\gamma}(\mathbf{s})+\rho_{\gamma}^{*}(P \backslash \mathbf{s})$, $l_{\gamma}^{(1)}\left(\mathbf{s} \cap P_{1}\right)=\rho_{\gamma}^{(1)}\left(\mathbf{s} \cap P_{1}\right)+\rho_{\gamma}^{*(1)}\left(P_{1} \backslash\left(\mathbf{s} \cap P_{1}\right)\right)$, and $l_{\gamma}^{(2)}\left(\mathbf{s} \cap P_{2}\right)=\rho_{\gamma}^{(2)}\left(\mathbf{s} \cap P_{2}\right)+\rho_{\gamma}^{*(2)}\left(P_{2} \backslash\left(\mathbf{s} \cap P_{2}\right)\right)$. Therefore $l_{\gamma}(\mathbf{s})=l_{\gamma}^{(1)}\left(\mathbf{s} \cap P_{1}\right)+l_{\gamma}^{(2)}\left(\mathbf{s} \cap P_{2}\right)$.

It can be shown that if either of the conditions on the maximal and minimal elements on $P_{1}$ and $P_{2}$ required for the statement " $P=P_{1} \triangleleft P_{2}$ " fail, then so does at least one of the decomposition equations in Lemma 3.1 for $\rho_{\gamma}(\mathbf{s})$ and $l_{\gamma}(\mathbf{s})$.

\section{4. $\mathfrak{g}$-semistandard posets, lattices, and tableaux}

We define special two-color grid posets $P$, the "g-semistandard posets". Then we define corresponding lattices $L=J_{\text {color }}(P)$, the "g-semistandard" lattices. In the second half of the section, "g-semistandard" tableau descriptions of the elements of these lattices are developed.

For the remainder of this paper, $\mathfrak{g}$ denotes a rank two semisimple Lie algebra: $\mathfrak{g} \in\left\{A_{1} \oplus\right.$ $\left.A_{1}, A_{2}, C_{2}, G_{2}\right\}$. We identify $\alpha$ with a short simple root for $\mathfrak{g}$ and $\beta$ as the other simple root. The vertex colors for the posets and the edge colors for the lattices which we now introduce correspond to the simple roots of $\mathfrak{g}$. So here the index set $I$ of Section 2 becomes $I=\{\alpha, \beta\}$. Let $\omega_{\alpha}=\omega_{1}=(1,0)$ and $\omega_{\beta}=\omega_{2}=(0,1)$ respectively denote the corresponding fundamental weights. Then any weight $\mu$ in $\Lambda$ of the form $\mu=p \omega_{\alpha}+q \omega_{\beta}$ (where $p$ and $q$ are integers) is now identified with the pair $(p, q)$ in $\mathbb{Z} \times \mathbb{Z}$. In particular, $\alpha$ and $\beta$ are respectively identified with the first and second row vectors from the Cartan matrix $M$ for $\mathfrak{g}$. These matrices, displayed in Figure 4.1, specify the $\mathfrak{g}$-structure condition of Section 2 for edge-colored ranked posets.

The $\mathfrak{g}$-fundamental posets $P_{\mathfrak{g}}(1,0)$ and $P_{\mathfrak{g}}(0,1)$ are defined to be the two-color grid posets of Figure 4.2. The corresponding $\mathfrak{g}$-fundamental lattices are defined to be the edge-colored lattices $L_{\mathfrak{g}}(1,0):=J_{\text {color }}\left(P_{\mathfrak{g}}(1,0)\right)$ and $L_{\mathfrak{g}}(0,1):=J_{\text {color }}\left(P_{\mathfrak{g}}(0,1)\right)$. See Figure 4.3. For the remainder of 


\begin{tabular}{|c|c|c|c|c|}
\hline Figure 4.1 & $\left(\begin{array}{ll}2 & 0 \\
0 & 2\end{array}\right)$ & $\left(\begin{array}{cc}2 & -1 \\
-1 & 2\end{array}\right)$ & $\left(\begin{array}{cc}2 & -1 \\
-2 & 2\end{array}\right)$ & $\left(\begin{array}{cc}2 & -1 \\
-3 & 2\end{array}\right)$ \\
\hline
\end{tabular}

Figure 4.2: $\mathfrak{g}$-fundamental posets.

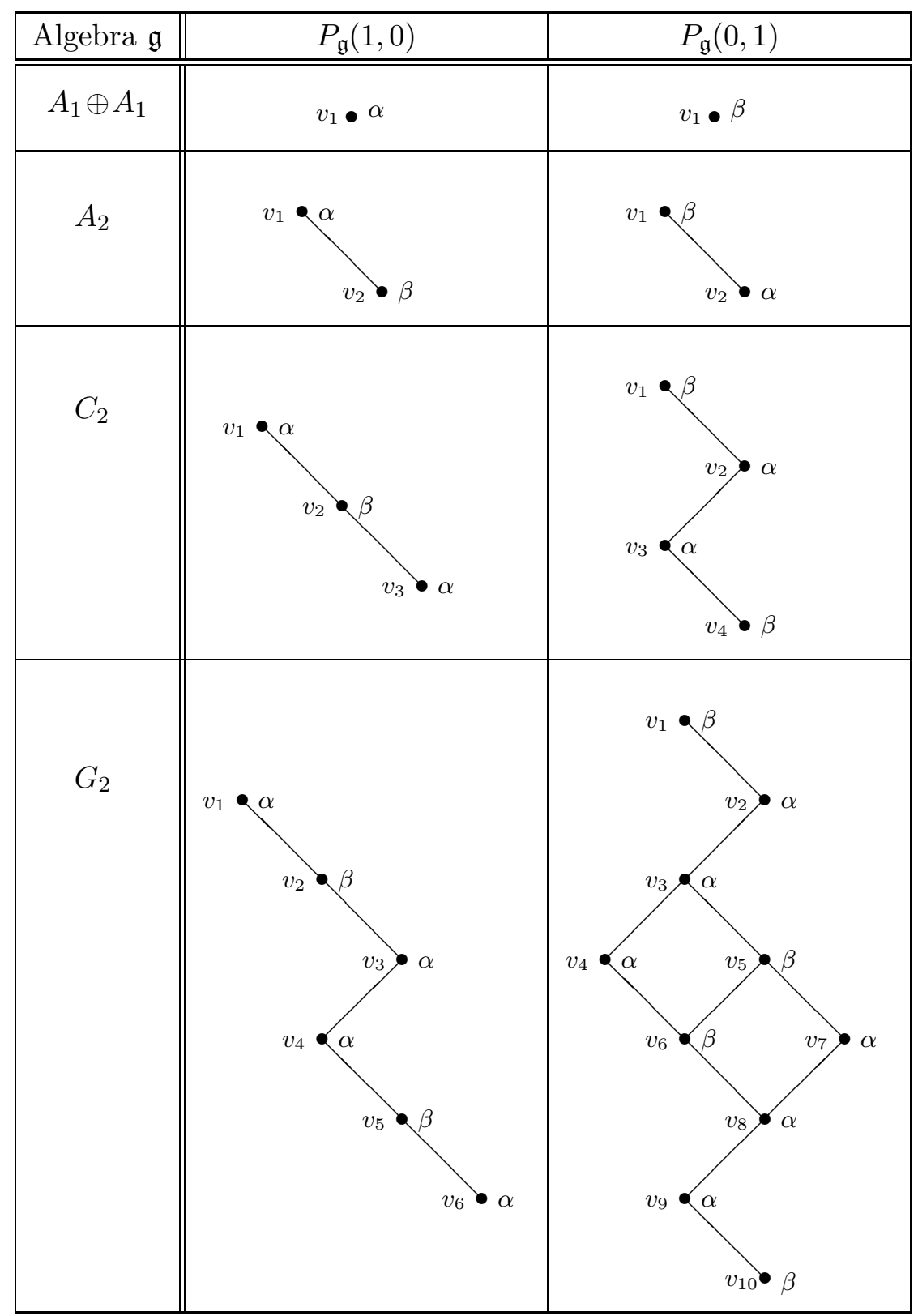

this section, everything presented for the simple cases $\left(A_{2}, C_{2}\right.$, and $\left.G_{2}\right)$ has an easy $A_{1} \oplus A_{1}$ analog. The details for $A_{1} \oplus A_{1}$ are omitted to save space, beginning with Figure 4.3.

Let $\lambda=(a, b)$, with $a, b \geq 0$. The $\mathfrak{g}$-semistandard poset $P_{\mathfrak{g}}^{\beta \alpha}(\lambda)$ associated to $\lambda$ is defined to be the two-color grid poset $P$ which has the decomposition $P_{1} \triangleleft P_{2} \triangleleft \cdots \triangleleft P_{a+b}$, where $P_{i}$ is vertex-color isomorphic to $P_{\mathfrak{g}}(0,1)$ for $1 \leq i \leq b$ and to $P_{\mathfrak{g}}(1,0)$ for $1+b \leq i \leq a+b$. It can be seen that $P$ is 
Figure 4.3: Elements of $\mathfrak{g}$-fundamental lattices as order ideals of $\mathfrak{g}$-fundamental posets.

(Each order ideal is identified by the indices of its maximal vertices. )

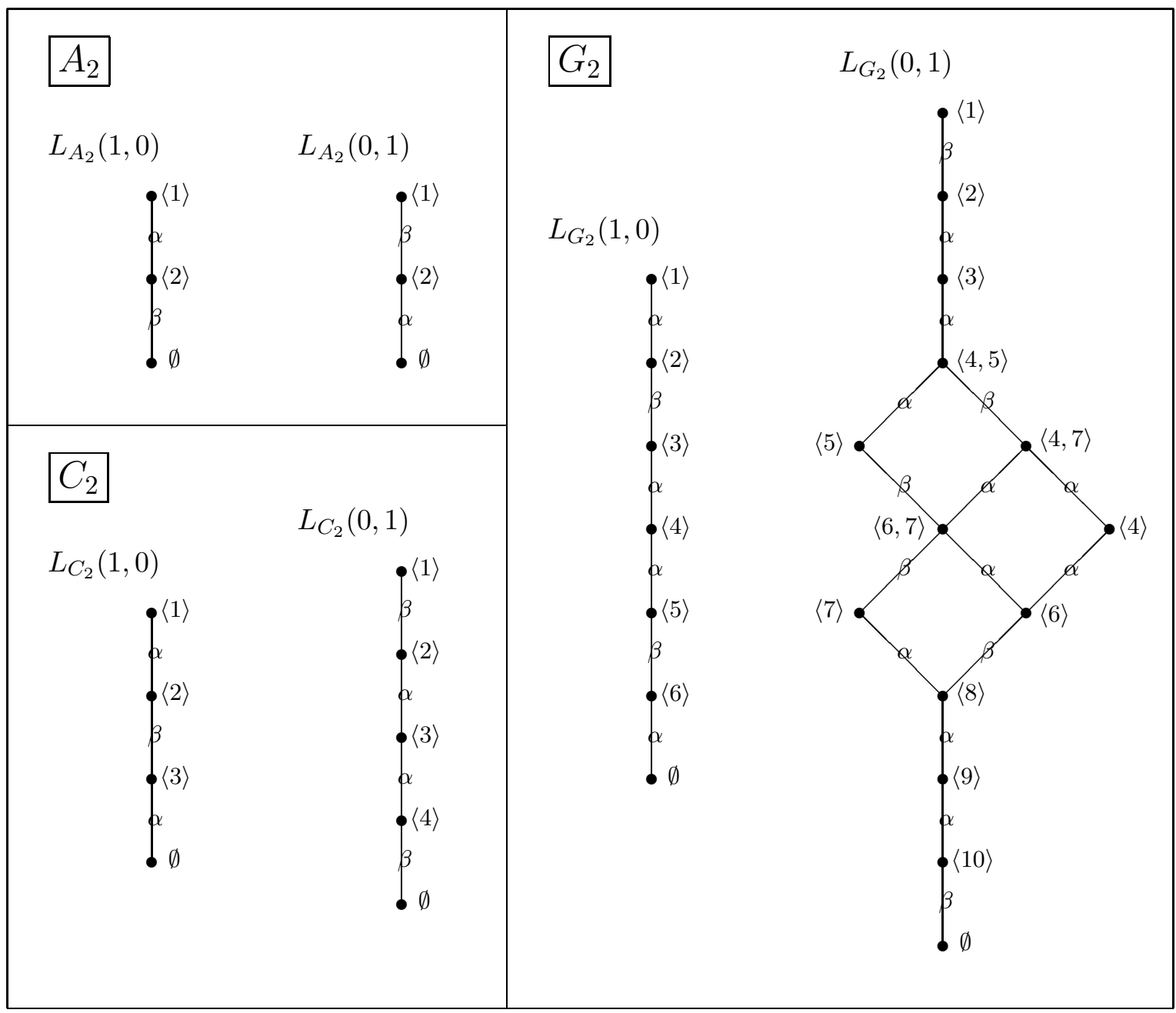

unique up to isomorphism. For each semisimple Lie algebra $\mathfrak{g}$, the poset $P_{\mathfrak{g}}^{\beta \alpha}(2,2)$ is depicted in Figure 3.2. The $\mathfrak{g}$-semistandard poset $P_{\mathfrak{g}}^{\alpha \beta}(\lambda)$ associated to $\lambda$ is analogously defined, except with $P_{i}$ vertex-color isomorphic to $P_{\mathfrak{g}}(1,0)$ for $1 \leq i \leq a$ and to $P_{\mathfrak{g}}(0,1)$ for $a+1 \leq i \leq a+b$. See Figure 3.3 for the corresponding $P_{\mathfrak{g}}^{\alpha \beta}(2,2)$. Note that $P_{\mathfrak{g}}^{\beta \alpha}(1,0)=P_{\mathfrak{g}}^{\alpha \beta}(1,0)=P_{\mathfrak{g}}(1,0)$, and $P_{\mathfrak{g}}^{\beta \alpha}(0,1)=P_{\mathfrak{g}}^{\alpha \beta}(0,1)=P_{\mathfrak{g}}(0,1)$. If $a=b=0$, then $P_{\mathfrak{g}}^{\beta \alpha}(\lambda)$ and $P_{\mathfrak{g}}^{\alpha \beta}(\lambda)$ are the empty set. The $\mathfrak{g}$-semistandard lattices associated to $\lambda$ are the edge-colored lattices $L_{\mathfrak{g}}^{\beta \alpha}(\lambda):=J_{\text {color }}\left(P_{\mathfrak{g}}^{\beta \alpha}(\lambda)\right)$ and $L_{\mathfrak{g}}^{\alpha \beta}(\lambda):=J_{\text {color }}\left(P_{\mathfrak{g}}^{\alpha \beta}(\lambda)\right)$. Note that $L_{\mathfrak{g}}^{\beta \alpha}(1,0)=L_{\mathfrak{g}}^{\alpha \beta}(1,0)=L_{\mathfrak{g}}(1,0)$, and $L_{\mathfrak{g}}^{\beta \alpha}(0,1)=L_{\mathfrak{g}}^{\alpha \beta}(0,1)=$ $L_{\mathfrak{g}}(0,1)$. We will not consider "mixed" concatenations, where some copies of $P_{\mathfrak{g}}(0,1)$ are interlaced amongst copies of $P_{\mathfrak{g}}(1,0)$. Any such concatenation will not have the max property, which is possessed by all of the $\mathfrak{g}$-semistandard posets.

Each $\mathfrak{g}$-semistandard lattice is an edge-colored poset. From now on we write $w t(\mathbf{s})$ for $w t_{L}(\mathbf{s})$ when $L$ is $\mathfrak{g}$-semistandard. Let $\mathbf{s} \in L$. Let $\gamma \in\{\alpha, \beta\}$. By definition, the $\gamma$-entry of the 2-tuple $w t(\mathbf{s})$ is the rank of $\mathbf{s}$ within the $\gamma$-colored connected component of $\mathbf{s}$ diminished by the depth of $\mathbf{s}$ in that component. 
Lemma 4.1 Let $\mathbf{s} \stackrel{\gamma}{\rightarrow} \mathbf{t}$ be an edge of color $\gamma \in\{\alpha, \beta\}$ in a $\mathfrak{g}$-fundamental lattice $L$. Then $w t(\mathbf{s})+\gamma=w t(\mathbf{t})$. Hence each $\mathfrak{g}$-fundamental lattice satisfies the $\mathfrak{g}$-structure condition.

Proof. Note that $\mathbf{s}$ and $\mathbf{t}$ are in the same $\gamma$-component. Since $\mathbf{t}$ covers $\mathbf{s}$ in this component, the $\gamma$-entry of $w t(\mathbf{t})$ is 2 more than the $\gamma$-entry of $w t(\mathbf{s})$. But adding the simple root $\gamma$ to $w t(\mathbf{s})$ adds 2 to the $\gamma$-entry of $w t(\mathbf{s})$, since $M_{\gamma, \gamma}=2$ always. Let $\gamma^{\prime}$ in $I$ be such that $\gamma^{\prime} \neq \gamma$. Using Figure 4.4 , one can quickly check by hand that the $\gamma^{\prime}$-entry of $w t(\mathbf{s})$ changes by $M_{\gamma, \gamma^{\prime}}$ or by $M_{\gamma^{\prime}, \gamma}$ (as appropriate) for each edge within each $\gamma$-component of a $\mathfrak{g}$-fundamental lattice.

Proposition 4.2 Let $\lambda=(a, b)$, with $a, b \geq 0$. Let $L$ be one of the $\mathfrak{g}$-semistandard lattices $L_{\mathfrak{g}}^{\beta \alpha}(\lambda)$ or $L_{\mathfrak{g}}^{\alpha \beta}(\lambda)$. Let $\mathbf{s} \stackrel{\gamma}{\rightarrow} \mathbf{t}$ be an edge of color $\gamma \in\{\alpha, \beta\}$ in $L$. Then $w t(\mathbf{s})+\gamma=w t(\mathbf{t})$, and hence $L$ satisfies the $\mathfrak{g}$-structure condition.

Proof. In light of Lemma 4.1, apply part (2) of Lemma 3.1.

Remark 4.3 If $\mathfrak{g}=A_{1} \oplus A_{1}$, then we have $P_{\mathfrak{g}}^{\beta \alpha}(\lambda) \cong P_{\mathfrak{g}}^{\alpha \beta}(\lambda)$ as vertex-colored posets: their Hasse diagrams are vertex-color isomorphic to $P_{\mathfrak{g}}^{\beta \alpha}(a, 0) \oplus P_{\mathfrak{g}}^{\beta \alpha}(0, b) \cong \mathbf{a} \oplus \mathbf{b}$. Hence $L_{\mathfrak{g}}^{\beta \alpha}(\lambda)$ and $L_{\mathfrak{g}}^{\alpha \beta}(\lambda)$ are edge-color isomorphic to $L_{\mathfrak{g}}^{\beta \alpha}(a, 0) \times L_{\mathfrak{g}}^{\beta \alpha}(0, b) \cong(\mathbf{a}+\mathbf{1}) \times(\mathbf{b}+\mathbf{1})$. For $\mathfrak{g}=C_{2}$ or $\mathfrak{g}=G_{2}$, observe that $P_{\mathfrak{g}}^{\alpha \beta}(\lambda)$ is vertex-color isomorphic to $\left(P_{\mathfrak{g}}^{\beta \alpha}(\lambda)\right)^{*}$, and thus $L_{\mathfrak{g}}^{\alpha \beta}(\lambda)$ and $\left(L_{\mathfrak{g}}^{\beta \alpha}(\lambda)\right)^{*}$ are isomorphic as edge-colored posets. For $\mathfrak{g}=A_{2}, P_{\mathfrak{g}}^{\alpha \beta}(\lambda)$ and $\left(P_{\mathfrak{g}}^{\beta \alpha}(\lambda)\right)^{*}$ are isomorphic as posets, but their vertex colors are reversed; disregarding edge colors, it follows that $L_{\mathfrak{g}}^{\alpha \beta}(\lambda)$ and $\left(L_{\mathfrak{g}}^{\beta \alpha}(\lambda)\right)^{*}$ are isomorphic as posets. In all cases, $L_{\mathfrak{g}}^{\alpha \beta}(\lambda) \cong\left(L_{\mathfrak{g}}^{\beta \alpha}(\lambda)\right)^{\triangle}$.

The easy proof of the following statement will be omitted:

Lemma 4.4 Let $\lambda=(a, b)$, with $a, b \geq 0$. If $\mathfrak{g}$ is simple, then $L_{\mathfrak{g}}^{\beta \alpha}(\lambda) \cong L_{\mathfrak{g}}^{\alpha \beta}(\lambda)$ as edge-colored posets if and only if $a=0$ or $b=0$.

Now we develop tableau labels for the elements of half of the $\mathfrak{g}$-semistandard lattices, the $L_{\mathfrak{g}}^{\beta \alpha}(\lambda)$. Comments relating these tableaux to tableaux developed by some of us and other authors appear in Section 5. We associate to the fundamental weight $\omega_{\alpha}=(1,0)$ the shape shape $(1,0)=\square$; we associate to $\omega_{\beta}=(0,1)$ the shape $\operatorname{shape}(0,1)=\square$. For $a, b \geq 0$, we associate to $\lambda=(a, b)$ the shape (Ferrers diagram) with $b$ columns of length two and $a$ columns of length one. A tableau of shape $\lambda$ is a filling of the boxes of shape $(\lambda)$ with entries from some totally ordered set. For a tableau $T$ of shape $\lambda$, we write $T=\left(T^{(1)}, \ldots, T^{(a+b)}\right)$, where $T^{(i)}$ is the $i$ th column of $T$ from the left. We let $T_{j}^{(i)}$ denote the $j$ th entry of the column $T^{(i)}$, counting from the top. The tableau $T$ is semistandard if the entries weakly increase across rows and strictly increase down columns. To each element $\mathbf{t}$ of a $\mathfrak{g}$-fundamental lattice from Figure 4.3 we associate the one-column semistandard tableau tableau(t) of Figure 4.4. For an order ideal $\mathbf{t}$ of $P_{\mathfrak{g}}^{\beta \alpha}(\lambda)$, let tableau(t) be the tableau $T=\left(T^{(1)}, \ldots, T^{(a+b)}\right)$ with $T^{(i)}=\operatorname{tableau}\left(\mathbf{t} \cap P_{i}\right)$. A tableau $T$ of shape $\lambda$ obtained in this way is a $\mathfrak{g}$-semistandard tableau of shape $\lambda$. We let $\mathcal{S}_{\mathfrak{g}}(\lambda)$ denote the set of all $\mathfrak{g}$-semistandard tableaux of shape $\lambda$. The function tableau : $L_{\mathfrak{g}}^{\beta \alpha}(\lambda) \longrightarrow \mathcal{S}_{\mathfrak{g}}(\lambda)$ is a one-to-one correspondence. See Figure 6.1 for a $C_{2}$ example.

Proposition 4.5 Let $a, b \geq 0$, and let $\lambda=(a, b)$. Then:

$\mathcal{S}_{A_{2}}(\lambda)=\{$ semistandard tableau $T$ of shape $\lambda$ with entries from $\{1,2,3\}\}$ 
Figure 4.4: Weights and tableaux for $\mathfrak{g}$-fundamental lattices.

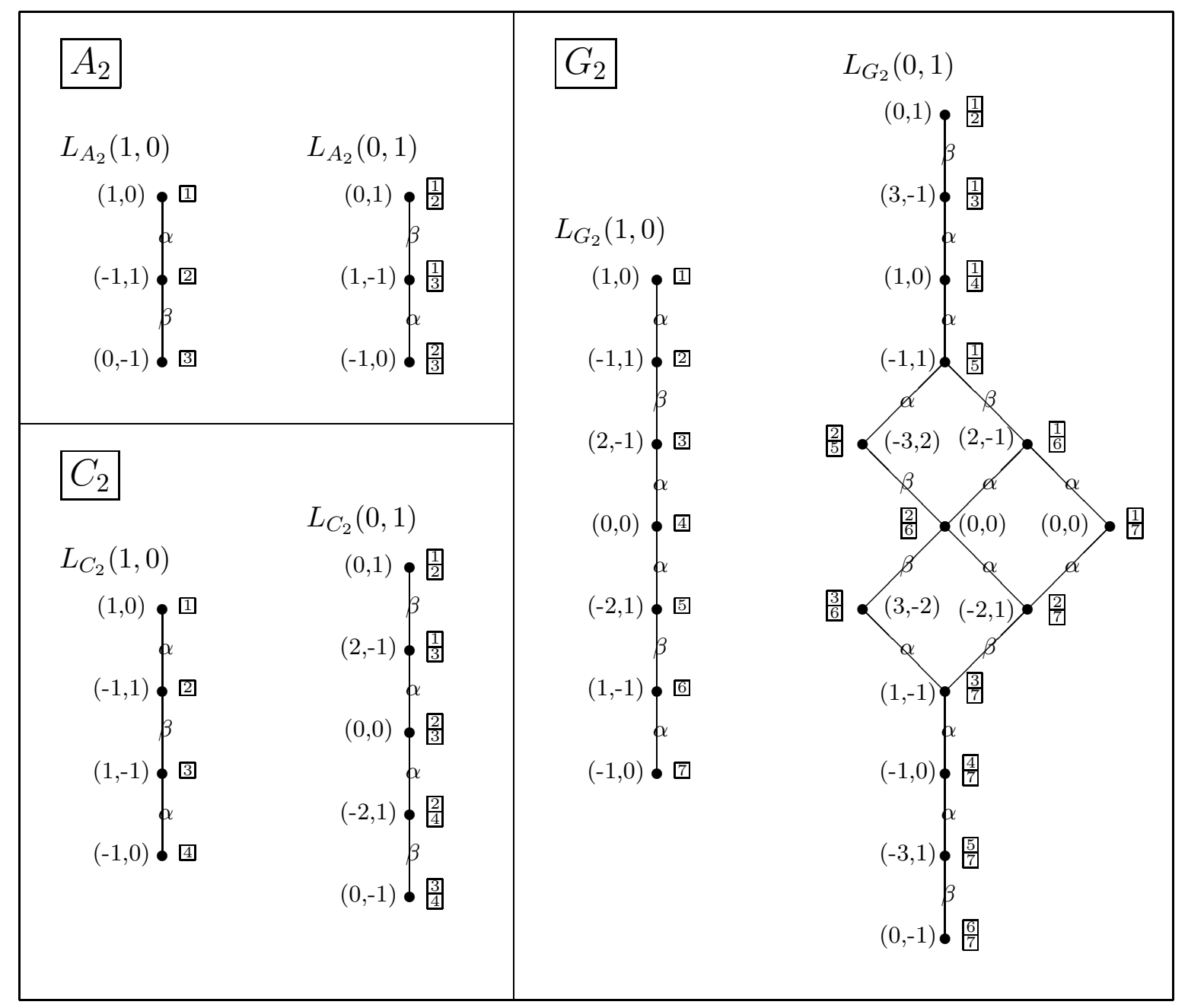

$\mathcal{S}_{C_{2}}(\lambda)=\{$ semistandard tableau $T$ of shape $\lambda$ with entries from $\{1,2,3,4\}$

$\frac{1}{4}$ is not a column of $T$, and $\frac{2}{3}$ appears at most once in $\left.T\right\}$

$\mathcal{S}_{G_{2}}(\lambda)=\{$ semistandard tableau $T$ of shape $\lambda$ with entries from $\{1,2,3,4,5,6,7\}$ the column 4 appears at most once in $T ; \frac{2}{3}, \frac{2}{4}, \frac{3}{4}, \frac{3}{5}, \frac{4}{5}, \frac{4}{6}$, and $\frac{5}{6}$ are not columns of $T$; plus the restrictions of Figure 4.5$\}$

Proof. The association of one-column $\mathfrak{g}$-semistandard tableaux with order ideals of $\mathfrak{g}$-fundamental posets is given in Figures 4.3 and 4.4. Consider the $\mathfrak{g}=C_{2}$ case. We want to show that the set $\mathcal{S}_{C_{2}}(\lambda)$ is the same as the stated set, which we denote $\mathcal{S}$. Let $T \in \mathcal{S}_{C_{2}}(\lambda)$, so $T=$ tableau $(\mathbf{t})$ for some order ideal $\mathbf{t}$ of $P_{C_{2}}^{\beta \alpha}(\lambda)$. Write $P_{C_{2}}^{\beta \alpha}(\lambda)=P_{1} \triangleleft \cdots \triangleleft P_{a+b}$, as depicted in Figure 4.6. Following Figure 4.2, we label the vertices of $P_{j}$ as $w_{1, j}, w_{2, j}, w_{3, j}$, and $w_{4, j}$ with $w_{1, j}>w_{2, j}>w_{3, j}>w_{4, j}$ whenever $1 \leq j \leq b$, and we label the vertices of $P_{j}$ as $z_{1, j}, z_{2, j}$, and $z_{3, j}$ with $z_{1, j}>z_{2, j}>z_{3, j}$ whenever $1+b \leq j \leq a+b$. By definition, $T=\left(T^{(1)}, \ldots, T^{(a+b)}\right)$ with $T^{(i)}=\operatorname{tableau}\left(\mathbf{t} \cap P_{i}\right)$. The entries for $T^{(i)}$ are from the set $\{1,2,3,4\}$, and no $T^{(i)}$ is the column $\frac{1}{4}$. To see how the 
Figure 4.5: Some restrictions for any given $G_{2}$-semistandard tableau $T$.

\begin{tabular}{|c|c|}
\hline Column $T^{(i)}$ of $T$ & Then the succeeding column $T^{(i+1)}$ of $T$ cannot be... \\
\hline 4 & 4 \\
\hline \begin{tabular}{|l|}
1 \\
4 \\
\end{tabular} & $1, \frac{1}{4}, \frac{1}{5}, \frac{1}{6}, \frac{1}{7}$ \\
\hline \begin{tabular}{|l|}
1 \\
5 \\
\end{tabular} & $1, \frac{1}{5}, \frac{1}{6}, \frac{1}{7}$ \\
\hline \begin{tabular}{|l|}
1 \\
6 \\
\end{tabular} & 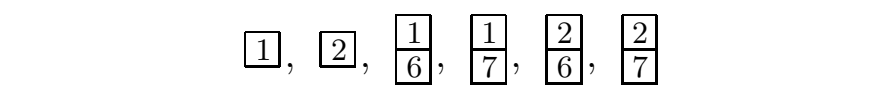 \\
\hline \begin{tabular}{|l|}
2 \\
6 \\
\end{tabular} & $2, \frac{2}{6}, \frac{2}{7}$ \\
\hline \begin{tabular}{|l|}
1 \\
7 \\
\end{tabular} & $1,2,3,4, \frac{1}{7}, \frac{2}{7}, \frac{3}{7}, \frac{4}{7}$ \\
\hline \begin{tabular}{|l|}
2 \\
7 \\
\end{tabular} & $2,3,4, \frac{2}{7}, \frac{3}{7}, \frac{4}{7}$ \\
\hline \begin{tabular}{|l|}
3 \\
7 \\
\end{tabular} & $3,4, \frac{3}{7}, \frac{4}{7}$ \\
\hline \begin{tabular}{|l|}
4 \\
7 \\
\end{tabular} & $4, \frac{4}{7}$ \\
\hline
\end{tabular}

semistandard and other restrictions occur, suppose (for example) that $T^{(i)}$ is the column $\frac{2}{3}$ for some $1 \leq i \leq b$. Note that $\mathbf{t} \cap P_{i}=\left\{w_{3, i}, w_{4, i}\right\}$. It follows that $w_{1, j}, w_{2, j}$, and $w_{3, j}$ are not in $\mathbf{t}$ for for $i<j \leq b$, and moreover $z_{1, j}$ is not in $\mathbf{t}$ for $1+b \leq j \leq a+b$. In particular, it follows that $T^{(i+1)}$ cannot be $\frac{1}{2}, \frac{1}{3}, \frac{2}{3}$, or 1 , assuming the column $T^{(i+1)}$ exists. That is, the pair of columns $T^{(i)}$ and $T^{(i+1)}$ meets the requirements for inclusion in the set $\mathcal{S}$. The other eight cases for $T^{(i)}$ can be handled in a similar fashion. We conclude that $T \in \mathcal{S}$. So $\mathcal{S}_{C_{2}}(\lambda) \subseteq \mathcal{S}$.

In the other direction, suppose $T=\left(T^{(1)}, \ldots, T^{(a+b)}\right)$ is in $\mathcal{S}$. For each $i$, let $Q_{i}$ be the order ideal of $P_{i}$ corresponding to the one-column tableau $T^{(i)}$, and let $\mathbf{t}:=\cup_{i} Q_{i}$. By examining cases as in the previous paragraph, one can check that the restrictions on $T$ as an element of $\mathcal{S}$ guarantee that $\mathbf{t}$ will be an order ideal of $P_{C_{2}}^{\beta \alpha}(\lambda)$ with $Q_{i}=\mathbf{t} \cap P_{i}$ for each $i$. Hence $T \in \mathcal{S}_{C_{2}}(\lambda)$. It follows that $\mathcal{S} \subseteq \mathcal{S}_{C_{2}}(\lambda)$, which completes the proof for the $C_{2}$ case. The $A_{2}$ and $G_{2}$ cases can be handled by similar arguments.

Remark 4.6 In passing we note that the partial ordering and the covering relations in $L_{\mathfrak{g}}^{\beta \alpha}(\lambda)$ are easy to describe with the "coordinates" of $\mathfrak{g}$-semistandard tableaux. For $\mathbf{s}$ and $\mathbf{t}$ in $L_{\mathfrak{g}}^{\beta \alpha}(\lambda)$, let $S:=\operatorname{tableau}(\mathbf{s})$ and $T:=\operatorname{tableau}(\mathbf{t})$. Then $\mathbf{s} \leq \mathbf{t}$ if and only if $S_{j}^{(i)} \geq T_{j}^{(i)}$ for all $i, j$. (This is the "reverse componentwise" order on tableaux.) Moreover, $\mathbf{s} \rightarrow \mathbf{t}$ is a covering relation in the poset $L_{\mathfrak{g}}^{\beta \alpha}(\lambda)$ if and only if for some $i$ and $j$ we have $S_{j}^{(i)}=T_{j}^{(i)}+1$ while $S_{q}^{(p)}=T_{q}^{(p)}$ for all $(p, q) \neq(i, j)$. For $\mathfrak{g}=A_{2}$, the edge gets color $\alpha$ if $T_{j}^{(i)}$ is 1 and color $\beta$ if $T_{j}^{(i)}$ is 2 ; for $\mathfrak{g}=C_{2}$, the edge gets color $\alpha$ if $T_{j}^{(i)}$ is 1 or 3 and color $\beta$ if $T_{j}^{(i)}$ is 2 ; for $\mathfrak{g}=G_{2}$, the edge gets color $\alpha$ if $T_{j}^{(i)}$ is 1 or 3 or 4 or 6 and color $\beta$ if $T_{j}^{(i)}$ is 2 or 5 . 
Figure 4.6: $P_{C_{2}}^{\beta \alpha}(\lambda)=P_{1} \triangleleft \cdots \triangleleft P_{a+b}$

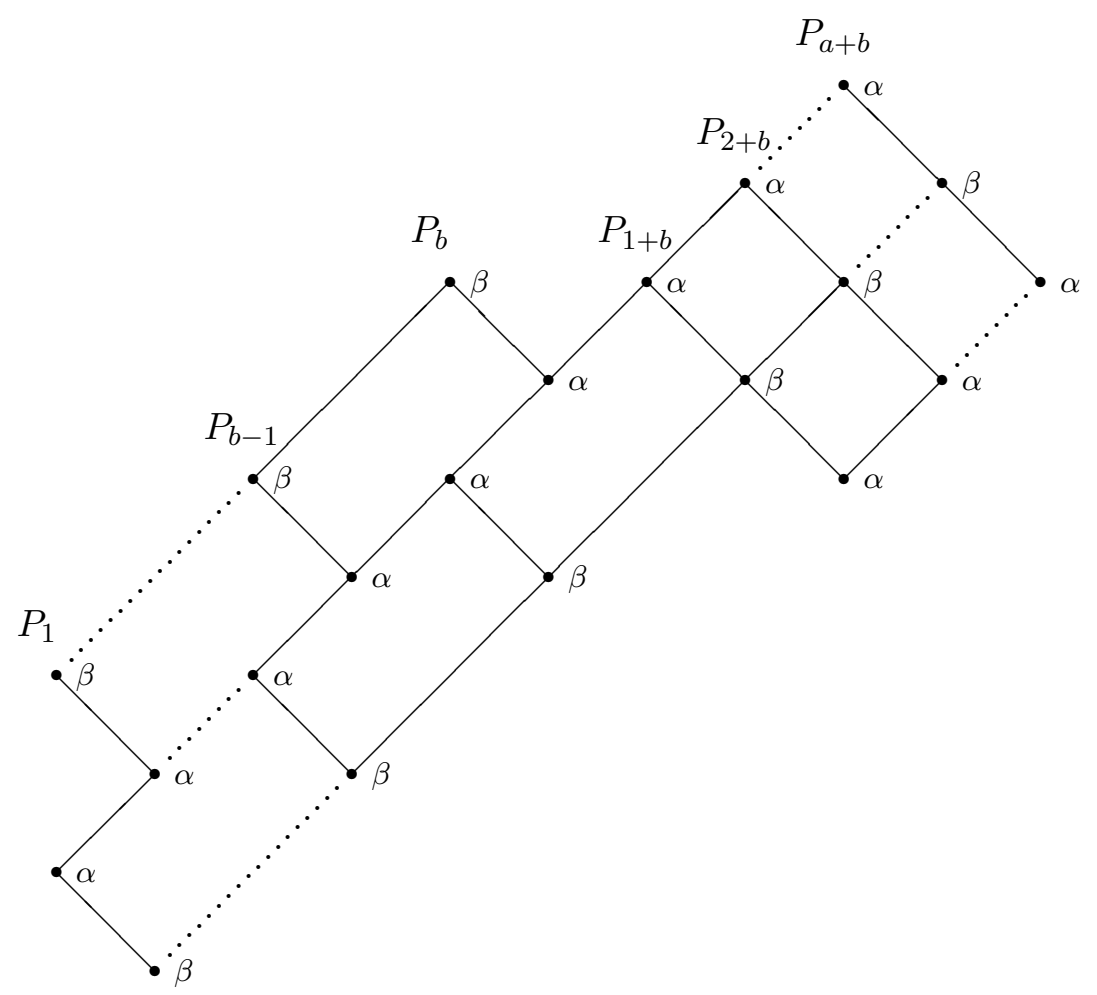

For a tableau $T$ of shape $\lambda=(a, b)$ and a positive integer $k$, we define $n_{k}(T)$ to be the number of times the entry $k$ appears in the tableau $T$. Observe that $n_{k}(T)=\sum_{i=1}^{a+b} n_{k}\left(T^{(i)}\right)$. Define a function tableauwt : $\mathcal{S}_{\mathfrak{g}}(\lambda) \longrightarrow \mathbb{Z} \times \mathbb{Z}$ by the rules:

$$
\operatorname{tableauwt}(T):= \begin{cases}\left(n_{1}(T)-n_{2}(T), n_{2}(T)-n_{3}(T)\right) & \text { if } \mathfrak{g}=A_{2} \\ \left.n_{1}(T)-n_{2}(T)+n_{3}(T)-n_{4}(T), n_{2}(T)-n_{3}(T)\right) & \text { if } \mathfrak{g}=C_{2} \\ n_{1}(T)-n_{2}(T)+2 n_{3}(T)-2 n_{5}(T)+n_{6}(T)-n_{7}(T), & \\ \left.n_{2}(T)-n_{3}(T)+n_{5}(T)-n_{6}(T)\right) & \text { if } \mathfrak{g}=G_{2}\end{cases}
$$

The function $w t(\mathbf{s})$ defined on $L_{\mathfrak{g}}^{\beta \alpha}(\lambda)$ in terms of the color components of $L_{\mathfrak{g}}^{\beta \alpha}(\lambda)$ can be expressed in terms of the tableau entry counts when the elements $\mathbf{s}$ of $L_{\mathfrak{g}}^{\beta \alpha}(\lambda)$ are viewed as tableaux $\mathbf{t}$ in $\mathcal{S}_{\mathfrak{g}}(\lambda)$ :

Proposition 4.7 Let $\lambda=(a, b)$, with $a, b \geq 0$. For $\mathbf{t} \in L_{\mathfrak{g}}^{\beta \alpha}(\lambda)$, consider $T:=\operatorname{tableau}(\mathbf{t}) \in \mathcal{S}_{\mathfrak{g}}(\lambda)$. Then $w t(\mathbf{t})=$ tableauwt $(T)$.

Proof. With the help of Figures 4.4 and 4.5, one can easily confirm the result by hand whenever $\lambda$ is a fundamental weight. Then more generally apply Lemma 3.1 to $w t(\mathbf{t})$, noting that $\operatorname{tableauwt}(T)=\sum_{i=1}^{a+b} \operatorname{tableauwt}\left(T^{(i)}\right)$.

This concludes our self-contained development of $\mathfrak{g}$-semistandard posets, $\mathfrak{g}$-semistandard lattices, and $\mathfrak{g}$-semistandard tableaux in Sections 3 and 4 . 


\section{Weyl characters; Littelmann's tableaux; main results}

Our main result, Theorem 5.3, expresses the Weyl characters for the irreducible representations of the rank two semisimple Lie algebras as generating functions for $\mathfrak{g}$-semistandard lattices. We begin by recording some explicit data on roots, weights, Weyl groups, and irreducible characters for the rank two semisimple Lie algebras. Then we describe certain tableaux obtained by Littelmann in [Lit], and in Proposition 5.2 we match these with our $\mathfrak{g}$-semistandard tableaux. Corollary 5.4 gives the product expressions for the rank generating functions.

In rank two we denote the elements $e_{\omega_{\alpha}}$ and $e_{\omega_{\beta}}$ of the group ring $\mathbb{Z}[\Lambda]$ by $x$ and $y$. The reflections $s_{\alpha}$ and $s_{\beta}$ in $W$ act on the fundamental weights as follows: $s_{\alpha} \omega_{\alpha}=\omega_{\alpha}-\alpha, s_{\alpha} \omega_{\beta}=\omega_{\beta}, s_{\beta} \omega_{\alpha}=\omega_{\alpha}$, and $s_{\beta} \omega_{\beta}=\omega_{\beta}-\beta$. Figure 5.1 has data for the simple roots, positive roots, and Weyl group for each of the rank two simple Lie algebras. Recall from Section 2 that the denominator $A_{\varrho}$ of the Weyl character formula can be expressed as a product over the positive roots. Also recall that the numerator $A_{\varrho+\lambda}$ is an alternating sum over the elements of the Weyl group. Using the data of Figure 5.1 one obtains for $A_{2}$

$$
\begin{aligned}
A_{\varrho}= & x y\left(1-x^{-2} y\right)\left(1-x y^{-2}\right)\left(1-x^{-1} y^{-1}\right) \\
= & x y-x^{-1} y^{2}-x^{2} y^{-1}+x^{-2} y+x y^{-2}-x^{-1} y^{-1} \\
A_{\varrho+\lambda}= & x^{a+1} y^{b+1}-x^{-(a+1)} y^{a+b+2}-x^{a+b+2} y^{-(b+1)} \\
& +x^{-(a+b+2)} y^{a+1}+x^{b+1} y^{-(a+b+2)}-x^{-(b+1)} y^{-(a+1)}
\end{aligned}
$$

For $C_{2}$ we get:

$$
\begin{aligned}
A_{\varrho}= & x y\left(1-x^{-2} y\right)\left(1-x^{2} y^{-2}\right)\left(1-y^{-1}\right)\left(1-x^{-2}\right) \\
= & x y-x^{-1} y^{2}-x^{3} y^{-1}+x^{-3} y^{2}+x^{3} y^{-2}-x^{-3} y^{1}-x y^{-2}+x^{-1} y^{-1} \\
A_{\varrho+\lambda}= & x^{a+1} y^{b+1}-x^{-(a+1)} y^{a+b+2}-x^{a+2 b+3} y^{-(b+1)}+x^{-(a+2 b+3)} y^{a+b+2} \\
& +x^{a+2 b+3} y^{-(a+b+2)}-x^{-(a+2 b+3)} y^{b+1}-x^{a+1} y^{-(a+b+2)}+x^{-(a+1)} y^{-(b+1)}
\end{aligned}
$$

And for $G_{2}$ we have:

$$
\begin{aligned}
A_{\varrho}= & x y\left(1-x^{-2} y\right)\left(1-x^{3} y^{-2}\right)\left(1-x y^{-1}\right)\left(1-x^{-1}\right)\left(1-x^{-3} y\right)\left(1-y^{-1}\right) \\
= & x y-x^{-1} y^{2}-x^{4} y^{-1}+x^{-4} y^{3}+x^{5} y^{-2}-x^{-5} y^{3}-x^{5} y^{-3}+x^{-5} y^{2}+x^{4} y^{-3} \\
& -x^{-4} y-x y^{-2}+x^{-1} y^{-1} \\
A_{\varrho+\lambda}= & x^{a+1} y^{b+1}-x^{-(a+1)} y^{a+b+2}-x^{a+3 b+4} y^{-(b+1)}+x^{-(a+3 b+4)} y^{a+2 b+3}+x^{2 a+3 b+5} y^{-(a+b+2)} \\
& -x^{-(2 a+3 b+5)} y^{a+2 b+3}-x^{2 a+3 b+5} y^{-(a+2 b+3)}+x^{-(2 a+3 b+5)} y^{a+b+2}+x^{a+3 b+4} y^{-(a+2 b+3)} \\
& -x^{-(a+3 b+4)} y^{b+1}-x^{a+1} y^{-(a+b+2)}+x^{-(a+1)} y^{-(b+1)}
\end{aligned}
$$

We now seek a correspondence between our $\mathfrak{g}$-semistandard tableaux and certain tableaux of Littelmann [Lit]. Littelmann's tableaux are "translations" of the standard monomial theory tableaux of Lakshmibai and Seshadri. The roles of his columns and rows are reversed with respect to this paper. We pre-process Littelmann's tableaux in two steps. First, we reflect them across the main 
Figure 5.1: Roots and Weyl groups for the rank two simple Lie algebras.

\begin{tabular}{|c|c|c|c|}
\hline Algebra & Simple roots & Positive roots & $\begin{array}{c}\text { Weyl group } W \\
\text { (By generators and relations; as reduced words) }\end{array}$ \\
\hline$A_{2}$ & $\begin{array}{c}\alpha=2 \omega_{\alpha}-\omega_{\beta} \\
\beta=-\omega_{\alpha}+2 \omega_{\beta}\end{array}$ & $\alpha, \beta, \alpha+\beta$ & $\begin{array}{c}\left\langle s_{\alpha}, s_{\beta} \mid s_{\alpha}^{2}=s_{\beta}^{2}=i d,\left(s_{\alpha} s_{\beta}\right)^{3}=i d\right\rangle \\
\left\{i d, s_{\alpha}, s_{\beta}, s_{\alpha} s_{\beta}, s_{\beta} s_{\alpha}, s_{\alpha} s_{\beta} s_{\alpha}=s_{\beta} s_{\alpha} s_{\beta}\right\}\end{array}$ \\
\hline$C_{2}$ & $\begin{array}{c}\alpha=2 \omega_{\alpha}-\omega_{\beta} \\
\beta=-2 \omega_{\alpha}+2 \omega_{\beta}\end{array}$ & $\begin{array}{c}\alpha, \beta, \alpha+\beta \\
2 \alpha+\beta\end{array}$ & $\begin{array}{c}\left\langle s_{\alpha}, s_{\beta} \mid s_{\alpha}^{2}=s_{\beta}^{2}=i d,\left(s_{\alpha} s_{\beta}\right)^{4}=i d\right\rangle \\
\left\{i d, s_{\alpha}, s_{\beta}, s_{\alpha} s_{\beta}, s_{\beta} s_{\alpha}, s_{\alpha} s_{\beta} s_{\alpha}, s_{\beta} s_{\alpha} s_{\beta}\right. \\
\left.s_{\alpha} s_{\beta} s_{\alpha} s_{\beta}=s_{\beta} s_{\alpha} s_{\beta} s_{\alpha}\right\}\end{array}$ \\
\hline$G_{2}$ & $\begin{array}{c}\alpha=2 \omega_{\alpha}-\omega_{\beta} \\
\beta=-3 \omega_{\alpha}+2 \omega_{\beta}\end{array}$ & $\begin{array}{c}\alpha, \beta, \alpha+\beta \\
2 \alpha+\beta \\
3 \alpha+\beta \\
3 \alpha+2 \beta\end{array}$ & $\begin{array}{c}\left\langle s_{\alpha}, s_{\beta} \mid s_{\alpha}^{2}=s_{\beta}^{2}=i d,\left(s_{\alpha} s_{\beta}\right)^{6}=i d\right\rangle \\
\left\{i d, s_{\alpha}, s_{\beta}, s_{\alpha} s_{\beta}, s_{\beta} s_{\alpha}, s_{\alpha} s_{\beta} s_{\alpha}, s_{\beta} s_{\alpha} s_{\beta}\right. \\
s_{\alpha} s_{\beta} s_{\alpha} s_{\beta}, s_{\beta} s_{\alpha} s_{\beta} s_{\alpha}, s_{\alpha} s_{\beta} s_{\alpha} s_{\beta} s_{\alpha} \\
\left.s_{\beta} s_{\alpha} s_{\beta} s_{\alpha} s_{\beta}, s_{\alpha} s_{\beta} s_{\alpha} s_{\beta} s_{\alpha} s_{\beta}=s_{\beta} s_{\alpha} s_{\beta} s_{\alpha} s_{\beta} s_{\alpha}\right\}\end{array}$ \\
\hline
\end{tabular}

diagonal $i=j$. Then we group $k$ of his columns at a time into a "block" of $k$ columns, where $k=1$ for $A_{2}, k=2$ for $C_{2}$, and $k=6$ for $G_{2}$. We define $\operatorname{shape}(k \times \lambda):=\operatorname{shape}(\mu)$, where $\mu=k a \omega_{\alpha}+k b \omega_{\beta}=(k a, k b)$. A $k$-tableau of shape $\lambda$ is a filling of $\operatorname{shape}(k \times \lambda)$ with entries from some totally ordered set. The semistandard condition on $k$-tableaux is the same as the semistandard condition of Section 4. For a $k$-tableau $T$ of shape $\lambda$, we write $T=\left(T^{(1)}, \ldots, T^{(a+b)}\right)$, where $T^{(i)}$ is the $i$ th block of $k$ columns of $T$ counting from the left. Only certain fillings of these $k$-column blocks will be "admissible". Here are our processed versions of Littelmann's tableaux:

Definition 5.1 Let $\lambda=(a, b)$, with $a, b \geq 0$. Then:

$$
\begin{aligned}
& \mathcal{L} \mathcal{T}_{A_{2}}(\lambda):=\left\{\begin{array}{r}
\text { semistandard } 1 \text {-tableau } T \text { of shape } \lambda \text { with entries from }\{1,2,3\} \\
\text { admissible 1-column blocks of } T \text { come from Figure } 5.2\}
\end{array}\right. \\
& \mathcal{L} \mathcal{T}_{C_{2}}(\lambda):=\left\{\begin{array}{r}
\text { semistandard 2-tableau } T \text { of shape } \lambda \text { with entries from }\{1,2,3,4\} \\
\text { admissible 2-column blocks of } T \text { come from Figure } 5.2\}
\end{array}\right. \\
& \mathcal{L} \mathcal{T}_{G_{2}}(\lambda):=\left\{\begin{array}{r}
\text { semistandard } 6 \text {-tableau } T \text { of shape } \lambda \text { with entries from }\{1,2,3,4,5,6\} \\
\text { admissible } 6 \text {-column blocks of } T \text { come from Figure } 5.2\}
\end{array}\right.
\end{aligned}
$$

Moreover, the weight $w t_{\text {Lit }}(T)$ of a Littelmann tableau $T$ is given by

$$
w t_{L i t}(T):=\left\{\begin{array}{cc}
\left(n_{1}(T)-n_{2}(T)\right) \omega_{\alpha}+\left(n_{2}(T)-n_{3}(T)\right) \omega_{\beta} & \text { if } \mathfrak{g}=A_{2} \\
\frac{1}{2}\left[\left(n_{1}(T)-n_{2}(T)+n_{3}(T)-n_{4}(T)\right) \omega_{\alpha}+\left(n_{2}(T)-n_{3}(T)\right) \omega_{\beta}\right] & \text { if } \mathfrak{g}=C_{2} \\
\frac{1}{6}\left[\left(n_{1}(T)-n_{2}(T)+2 n_{3}(T)-2 n_{4}(T)+n_{5}(T)-n_{6}(T)\right) \omega_{\alpha}\right. & \\
\left.+\left(n_{2}(T)-n_{3}(T)+n_{4}(T)-n_{5}(T)\right) \omega_{\beta}\right] & \text { if } \mathfrak{g}=G_{2}
\end{array}\right.
$$


To obtain these tableaux for $A_{2}$, see Section 2 of [Lit]; for $C_{2}$, see the Appendix of [Lit]; and for $G_{2}$ see Section 3 of that paper. Littelmann expresses his weight function in terms of a basis $\left\{\varepsilon_{1}, \varepsilon_{2}\right\}$ for $\Lambda$, where $\varepsilon_{1}=\omega_{\alpha}$ and $\varepsilon_{2}=\omega_{\beta}-\omega_{\alpha}$. A consequence of standard monomial theory is: Theorem (Littelmann, Lakshmibai, Seshadri) Let $\lambda=(a, b)$, with $a, b \geq 0$. Let $\mathfrak{g}$ be a rank two simple Lie algebra. Then $\left(\mathcal{L} \mathcal{T}_{\mathfrak{g}}(\lambda), w t_{\text {Lit }}\right)$ is a splitting system for the irreducible character $\chi_{\lambda}$.

Next we describe a weight-preserving bijection $\phi: \mathcal{S}_{\mathfrak{g}}(\lambda) \longrightarrow \mathcal{L} \mathcal{T}_{\mathfrak{g}}(\lambda)$. For fundamental weights, the correspondence between the $\mathfrak{g}$-semistandard tableaux of Section 4 and Littelmann $k$-tableaux of this section is given in Figure 5.2. Given a rank two simple Lie algebra $\mathfrak{g}$, a dominant weight $\lambda=a \omega_{\alpha}+b \omega_{\beta}=(a, b)$, and a tableau $T$ in $\mathcal{S}_{\mathfrak{g}}(\lambda)$, we let $U=\phi(T)$ be the Littelmann $k$-tableau of shape $\lambda$ whose $i$ th $k$-column block $U^{(i)}$ corresponds to the $i$ th column $T^{(i)}$ of $T$. Keeping in mind the restrictions on which columns can follow $T^{(i)}$ to form a $\mathfrak{g}$-semistandard tableau $T$ in $\mathcal{S}_{\mathfrak{g}}(\lambda)$, one can check that $U^{(i)}$ followed by $U^{(i+1)}$ obeys the semistandard requirement for Littelmann $k$-tableaux. Hence $U$ is in $\mathcal{L} \mathcal{T}_{\mathfrak{g}}(\lambda)$. Similarly, given $U$ in $\mathcal{L} \mathcal{T}_{\mathfrak{g}}(\lambda)$, let $T=\psi(U)$ be the tableau of shape $\lambda$ whose $i$ th column $T^{(i)}$ corresponds to the $i$ th $k$-column block $U^{(i)}$ of $U$. Keeping in mind the semistandard condition on the Littelmann $k$-tableaux in $\mathcal{L} \mathcal{T}_{\mathfrak{g}}(\lambda)$, one can check that $T^{(i)}$ followed by $T^{(i+1)}$ obeys the restrictions for $\mathfrak{g}$-semistandard tableaux in $\mathcal{S}_{\mathfrak{g}}(\lambda)$, and hence $T$ is in $\mathcal{S}_{\mathfrak{g}}(\lambda)$. Clearly the mappings $\phi$ and $\psi$ are inverses.

Proposition 5.2 Keep the notation of the previous paragraph. The mapping $\phi: \mathcal{S}_{\mathfrak{g}}(\lambda) \longrightarrow \mathcal{L T}_{\mathfrak{g}}(\lambda)$ described above is a weight-preserving bijection: for any $T \in \mathcal{S}_{\mathfrak{g}}(\lambda), w t_{\text {Lit }}(\phi(T))=$ tableauwt $(T)$.

Proof. We must check that $\phi$ is weight-preserving. If $\lambda$ is a fundamental weight, simply inspect Figure 5.2. If $\lambda$ is a dominant weight and $T$ is in $\mathcal{S}_{\mathfrak{g}}(\lambda)$, then tableauwt $(T)=\sum$ tableauwt $\left(T^{(i)}\right)=$ $\sum w t_{L i t}\left(\phi\left(T^{(i)}\right)\right)$. The characterization of $w t_{\text {Lit }}$ in Definition 5.1 implies that $w t_{L i t}(\phi(T))=$ $\sum w t_{L i t}\left(\phi\left(T^{(i)}\right)\right)$.

Theorem 5.3 Let $\mathfrak{g}$ be a semisimple Lie algebra of rank two. Let $\lambda=(a, b)$, with $a, b \geq 0$. Let $L$ be one of the $\mathfrak{g}$-semistandard lattices $L_{\mathfrak{g}}^{\beta \alpha}(\lambda)$ or $L_{\mathfrak{g}}^{\alpha \beta}(\lambda)$. Then $L$ is a splitting poset for an irreducible representation of $\mathfrak{g}$ with highest weight $\lambda$. In particular,

$$
\operatorname{char}_{\mathfrak{g}}(\lambda ; x, y)=\sum_{\mathbf{s} \in L}(x, y)^{w t(\mathbf{s})}
$$

Proof. Proposition 4.2 states that $L$ satisfies the $\mathfrak{g}$-structure condition. Suppose $\mathfrak{g}$ is simple. Since $\left(\mathcal{L} \mathcal{T}_{\mathfrak{g}}(\lambda), w t_{\text {Lit }}\right)$ is a splitting system for $\chi_{\lambda}$, it follows from Proposition 5.2 that $\left(\mathcal{S}_{\mathfrak{g}}(\lambda)\right.$, tableauwt $)$ is as well. From Proposition 4.7 it now follows that $\left(L_{\mathfrak{g}}^{\beta \alpha}(\lambda), w t\right)$ is a splitting system for $\chi_{\lambda}$. Since $L_{\mathfrak{g}}^{\alpha \beta}(\lambda) \cong\left(L_{\mathfrak{g}}^{\beta \alpha}(\lambda)\right)^{\triangle}$, then by Lemma 2.2 the result holds for $L_{\mathfrak{g}}^{\alpha \beta}(\lambda)$ as well. The case $A_{1} \oplus A_{1}$ can be handled by constructing the corresponding representation.

The main results of $[\mathrm{Mc}]$ and $[\mathrm{Alv}]$ were closely related to Theorem 5.3 for the cases of $G_{2}$ and $C_{2}$ respectively. For these rank two simple Lie algebras $\mathfrak{g}$, the lattices $L_{\mathfrak{g}}^{\beta \alpha}(\lambda)$ were obtained by taking natural partial orders on the corresponding $\mathfrak{g}$-semistandard tableaux of Section 4 , and case analysis arguments were used to show that the mapping $\phi$ preserves weights and that the $\mathfrak{g}$-structure condition is satisfied. However, $\mathfrak{g}$-semistandard posets did not arise in their approach. If one is willing to depend entirely upon [Lit], then in this manner one can obtain Propositions 4.7 and 4.2 
Figure 5.2: Admissible $k$-column blocks for Littelmann tableau, their weights, and their corresponding $\mathfrak{g}$-semistandard columns.

\begin{tabular}{|c|c|c|c|c|c|}
\hline & & & \\
\hline & & & $\begin{array}{c}C_{2} \\
\text { Admissible } \\
\text { 2-block } T\end{array}$ & $\begin{array}{l}\text { Weight } \\
w t_{L i t}(T)\end{array}$ & $\begin{array}{c}\text { Corresponding } \\
C_{2} \text {-semistandard } \\
\text { tableau }\end{array}$ \\
\hline \multirow{2}{*}{$\begin{array}{c}A_{2} \\
\text { Admissible } \\
\text { 1-block } T\end{array}$} & \multirow{2}{*}{$\begin{array}{l}\text { Weight } \\
w t_{L i t}(T)\end{array}$} & \multirow{2}{*}{$\begin{array}{c}\text { Corresponding } \\
A_{2} \text {-semistandard } \\
\text { tableau }\end{array}$} & \begin{tabular}{|l|l|}
1 & 1 \\
\end{tabular} & $\omega_{\alpha}$ & \begin{tabular}{|l|}
1 \\
\end{tabular} \\
\hline & & & \begin{tabular}{l|l}
2 & 2 \\
\end{tabular} & $-\omega_{\alpha}+\omega_{\beta}$ & \begin{tabular}{|l|}
2 \\
\end{tabular} \\
\hline \begin{tabular}{|l|}
1 \\
\end{tabular} & $\omega_{\alpha}$ & \begin{tabular}{|l|}
1 \\
\end{tabular} & \begin{tabular}{l|l}
3 & 3 \\
\end{tabular} & $\omega_{\alpha}-\omega_{\beta}$ & \begin{tabular}{|l|}
3 \\
\end{tabular} \\
\hline \begin{tabular}{|l|}
2 \\
\end{tabular} & $-\omega_{\alpha}+\omega_{\beta}$ & \begin{tabular}{|l|}
2 \\
\end{tabular} & \begin{tabular}{|l|l|}
4 & 4 \\
\end{tabular} & $-\omega_{\alpha}$ & \begin{tabular}{|l|}
4 \\
\end{tabular} \\
\hline 3 & $-\omega_{\beta}$ & 3 & \begin{tabular}{l|l|}
1 & 1 \\
\end{tabular} & \multirow{2}{*}{$\omega_{\beta}$} & 1 \\
\hline 7 & \multirow[b]{2}{*}{$\omega_{\beta}$} & 1 & \begin{tabular}{l|l}
2 & 2 \\
\end{tabular} & & 2 \\
\hline $\begin{array}{l}1 \\
2 \\
\end{array}$ & & $\begin{array}{l}1 \\
2 \\
\end{array}$ & \begin{tabular}{|l|l}
1 & 1 \\
\end{tabular} & \multirow{2}{*}{$2 \omega_{\alpha}-\omega_{\beta}$} & 1 \\
\hline 1 & \multirow{2}{*}{$\omega_{\alpha}-\omega_{\beta}$} & 1 & & & 3 \\
\hline 3 & & 3 & 1 & \multirow{2}{*}{$0 \omega_{\alpha}+0 \omega_{\beta}$} & 2 \\
\hline 2 & \multirow[b]{2}{*}{$-\omega_{\alpha}$} & 2 & & & \\
\hline \multirow[t]{4}{*}{3} & & 3 & 2 & \multirow{2}{*}{$-2 \omega_{\alpha}+\omega_{\beta}$} & 2 \\
\hline & & & & & \\
\hline & & & 3 & \multirow{2}{*}{$-\omega_{\beta}$} & 3 \\
\hline & & & 4 & & 4 \\
\hline
\end{tabular}

\begin{tabular}{|c|c|c|c|c|c|c|c|}
\hline \multicolumn{6}{|c|}{$\begin{array}{c}G_{2} \\
\text { Admissible } \\
\text { 6-block } T\end{array}$} & $\begin{array}{l}\text { Weight } \\
w t_{L i t}(T)\end{array}$ & $\begin{array}{c}\text { Corresponding } \\
G_{2} \text {-semistandard } \\
\text { tableau }\end{array}$ \\
\hline 1 & 1 & 1 & 1 & 1 & 1 & $\omega_{\alpha}$ & \begin{tabular}{|l|}
1 \\
\end{tabular} \\
\hline 2 & 2 & 2 & 2 & 2 & 2 & $-\omega_{\alpha}+\omega_{\beta}$ & \begin{tabular}{|l|}
2 \\
\end{tabular} \\
\hline \begin{tabular}{|l|}
3 \\
\end{tabular} & 3 & 3 & 3 & 3 & 3 & $2 \omega_{\alpha}-\omega_{\beta}$ & \begin{tabular}{|l|}
3 \\
\end{tabular} \\
\hline \begin{tabular}{|l|}
3 \\
\end{tabular} & 3 & 3 & 4 & 4 & 4 & $0 \omega_{\alpha}+0 \omega_{\beta}$ & \begin{tabular}{|l|}
4 \\
\end{tabular} \\
\hline 4 & 4 & 4 & 4 & 4 & 4 & $-2 \omega_{\alpha}+\omega_{\beta}$ & \begin{tabular}{|l|}
5 \\
\end{tabular} \\
\hline \begin{tabular}{|l|}
5 \\
\end{tabular} & 5 & 5 & 5 & 5 & 5 & $\omega_{\alpha}-\omega_{\beta}$ & \begin{tabular}{|l|}
6 \\
\end{tabular} \\
\hline 6 & 6 & 6 & 6 & 6 & 6 & $-\omega_{\alpha}$ & \begin{tabular}{|l|}
7 \\
\end{tabular} \\
\hline
\end{tabular}

from Proposition 5.2 and Littelmann's analog to Theorem 5.3 without using Lemma 3.1. But this approach would take at least as much (related) work and would not be as uniformly stated. 
Figure 5.2 (continued): Admissible $k$-column blocks for Littelmann tableaux, their weights, and their corresponding $\mathfrak{g}$-semistandard columns.

\begin{tabular}{|c|c|c|c|c|c|c|c|}
\hline \multicolumn{6}{|c|}{$\begin{array}{c}G_{2} \\
\text { Admissible } \\
\text { 6-block } T\end{array}$} & $\begin{array}{l}\text { Weight } \\
w t_{L i t}(T)\end{array}$ & $\begin{array}{c}\text { Corresponding } \\
G_{2 \text {-semistandard }} \\
\text { tableau }\end{array}$ \\
\hline 1 & 1 & 1 & 1 & 1 & 1 & \multirow{2}{*}{$\omega_{\beta}$} & 1 \\
\hline 2 & 2 & 2 & 2 & 2 & 2 & & 2 \\
\hline 1 & 1 & 1 & 1 & 1 & 1 & \multirow{2}{*}{$3 \omega_{\alpha}-\omega_{\beta}$} & 1 \\
\hline 3 & 3 & 3 & 3 & 3 & 3 & & 3 \\
\hline 1 & 1 & 1 & 1 & 2 & 2 & \multirow{2}{*}{$\omega_{\alpha}$} & 1 \\
\hline 3 & 3 & 3 & 3 & 4 & 4 & & 4 \\
\hline 1 & 1 & 2 & 2 & 2 & 2 & \multirow{2}{*}{$-\omega_{\alpha}+\omega_{\beta}$} & 1 \\
\hline 3 & 3 & 4 & 4 & 4 & 4 & & 5 \\
\hline 2 & 2 & 2 & 2 & 2 & 2 & \multirow{2}{*}{$-3 \omega_{\alpha}+2 \omega_{\beta}$} & 2 \\
\hline 4 & 4 & 4 & 4 & 4 & 4 & & 5 \\
\hline 1 & 1 & 2 & 3 & 3 & 3 & \multirow{2}{*}{$2 \omega_{\alpha}-\omega_{\beta}$} & 1 \\
\hline 3 & 3 & 4 & 5 & 5 & 5 & & 6 \\
\hline 2 & 2 & 2 & 3 & 3 & 3 & \multirow{2}{*}{$0 \omega_{\alpha}+0 \omega_{\beta}$} & 2 \\
\hline 4 & 4 & 4 & 5 & 5 & 5 & & 6 \\
\hline 1 & 1 & 2 & 3 & 4 & 4 & \multirow{2}{*}{$0 \omega_{\alpha}+0 \omega_{\beta}$} & 1 \\
\hline 3 & 3 & 4 & 5 & 6 & 6 & & 7 \\
\hline 3 & 3 & 3 & 3 & 3 & 3 & \multirow{2}{*}{$3 \omega_{\alpha}-2 \omega_{\beta}$} & 3 \\
\hline 5 & 5 & 5 & 5 & 5 & 5 & & 6 \\
\hline 2 & 2 & 2 & 3 & 4 & 4 & \multirow{2}{*}{$-2 \omega_{\alpha}+\omega_{\beta}$} & 2 \\
\hline 4 & 4 & 4 & 5 & 6 & 6 & & 7 \\
\hline 3 & 3 & 3 & 3 & 4 & 4 & \multirow{2}{*}{$\omega_{\alpha}-\omega_{\beta}$} & 3 \\
\hline 5 & 5 & 5 & 5 & 6 & 6 & & 7 \\
\hline 3 & 3 & 4 & 4 & 4 & 4 & \multirow{2}{*}{$-\omega_{\alpha}$} & 4 \\
\hline 5 & 5 & 6 & 6 & 6 & 6 & & 7 \\
\hline 4 & 4 & 4 & 4 & 4 & 4 & \multirow{2}{*}{$-3 \omega_{\alpha}+\omega_{\beta}$} & 5 \\
\hline 6 & 6 & 6 & 6 & 6 & 6 & & 7 \\
\hline 5 & 5 & 5 & 5 & 5 & 5 & \multirow{2}{*}{$-\omega_{\beta}$} & 6 \\
\hline 6 & 6 & 6 & 6 & 6 & 6 & & 7 \\
\hline
\end{tabular}

Our final result presents the $\mathfrak{g}$-semistandard lattices as answers to Stanley's Problem 3 [Sta1]: Corollary 5.4 Let $\mathfrak{g}$ be a simple Lie algebra of rank two. Let $\lambda=(a, b)$, with $a, b \geq 0$. Then the $\mathfrak{g}$-semistandard lattices $L_{\mathfrak{g}}^{\beta \alpha}(\lambda)$ and $L_{\mathfrak{g}}^{\alpha \beta}(\lambda)$ are rank symmetric and rank unimodal. Moreover, the 
rank generating functions for these lattices are:

$$
\begin{gathered}
R G F_{A_{2}}(\lambda, q)=\frac{\left(1-q^{a+1}\right)\left(1-q^{b+1}\right)\left(1-q^{a+b+2}\right)}{(1-q)(1-q)\left(1-q^{2}\right)} \\
R G F_{C_{2}}(\lambda, q)=\frac{\left(1-q^{a+1}\right)\left(1-q^{b+1}\right)\left(1-q^{a+b+2}\right)\left(1-q^{a+2 b+3}\right)}{(1-q)(1-q)\left(1-q^{2}\right)\left(1-q^{3}\right)} \\
R G F_{G_{2}}(\lambda, q)=\frac{\left(1-q^{a+1}\right)\left(1-q^{b+1}\right)\left(1-q^{a+b+2}\right)\left(1-q^{a+2 b+3}\right)\left(1-q^{a+3 b+4}\right)\left(1-q^{2 a+3 b+5}\right)}{(1-q)(1-q)\left(1-q^{2}\right)\left(1-q^{3}\right)\left(1-q^{4}\right)\left(1-q^{5}\right)}
\end{gathered}
$$

In each case $\left|L_{\mathfrak{g}}^{\beta \alpha}(\lambda)\right|=\left|L_{\mathfrak{g}}^{\alpha \beta}(\lambda)\right|$, and these counts may be found by letting $q \rightarrow 1$.

Proof. In light of Theorem 5.3, apply Proposition 2.4. We have specialized the right hand side quotient there using the data from Figure 5.1.

\section{Remarks}

Stanley's Exercises 4.25 and 3.27 on Gaussian and pleasant posets have attracted some attention [Sta2]. A poset $P$ with $p$ elements is Gaussian if there exist positive integers $h_{1}, \ldots, h_{p}$ such that for all $m \geq 0$, the rank generating function of the lattice $J(P \times \mathbf{m})$ is $\Pi_{i=1}^{p}\left(1-q^{m+h_{i}}\right) /\left(1-q^{h_{i}}\right)$. In [Pr1], the sixth author and Stanley gave a uniform proof of the Gaussian property for all Gaussian posets. That proof used an analog of Theorem 5.3; it was based upon Seshadri's standard monomial basis theorem for the irreducible representations $X_{n}\left(m \omega_{k}\right)$, where the representations $X_{n}\left(\omega_{k}\right)$ are "minuscule". Now let $P$ be our $G_{2}$-fundamental poset $P_{G_{2}}(0,1)$ of Figure 4.2. Please use Figure 3.2 to help visualize the $G_{2}$-semistandard poset $P_{G_{2}}^{\beta_{\alpha}}(0, m)$ for $m \geq 0$. Note that $P_{G_{2}}^{\beta \alpha}(0, m)$ consists of $P \times \mathbf{m}$ together with some additional order relations. By Corollary 5.4, the rank generating function for $L_{G_{2}}^{\beta \alpha}(0, m)=J_{\text {color }}\left(P_{G_{2}}^{\beta \alpha}(0, m)\right)$ is

$$
\frac{\left(1-q^{m+1}\right)\left(1-q^{m+2}\right)\left(1-q^{2 m+3}\right)\left(1-q^{3 m+4}\right)\left(1-q^{3 m+5}\right)}{\left(1-q^{1}\right)\left(1-q^{2}\right)\left(1-q^{3}\right)\left(1-q^{4}\right)\left(1-q^{5}\right)} .
$$

One could introduce a more general notion of "quasi-Gaussian" for a poset $P$ by requiring that the elements of $P \times \mathbf{m}$ remain distinct when some additional (if any) order relations are introduced, and by allowing a more general product form for the generating function identity. Then the fundamental posets $P_{C_{2}}(0,1)$ and $P_{G_{2}}(1,0)$ of Figure 4.2 would also be quasi-Gaussian, but not Gaussian. In DW] more will be said about the order relations added to $P \times \mathbf{m}$ above and the juxtaposition rules for the fundamental posets shown in Figures 3.2 and 3.3. For now, we note that these added order relations are similar to those added in the following example: The "Catalan" poset $P_{3}$ of Figure 1.1 can be obtained by adding order relations to the Gaussian poset $\mathbf{3} \times \mathbf{3}$; this corresponds to the restriction of $\mathfrak{s l}_{6}\left(\omega_{3}\right)$ to $\mathfrak{s p}_{6}\left(\omega_{3}\right)$.

Here is the $C_{2}$ example promised in the middle of Section 4: The $C_{2}$-semistandard poset $P_{C_{2}}^{\beta \alpha}(1,1)$ is displayed in Figure 6.1. Also displayed is the corresponding $C_{2}$-semistandard lattice $L_{C_{2}}^{\beta \alpha}(1,1)$, with vertices labelled by the $C_{2}$-semistandard tableaux of shape $(1,1)$. The lattice $L_{C_{2}}^{\beta \alpha}(1,1)$ shown in Figure 6.1 looks similar in structure to the edge-colored lattice $L$ displayed in Figure 6.2. In fact, this $L=J_{\text {color }}(P)$ for the two-color grid poset $P$ displayed in Figure 6.2. Moreover, $P=Q_{1} \triangleleft Q_{2}$ with $Q_{1} \cong P_{1}$ and $Q_{2} \cong P_{2}$ for the indecomposable two-color grid posets $P_{1}$ and $P_{2}$ displayed in 
Figure 6.1: $P_{C_{2}}^{\beta \alpha}(1,1)$ and $L_{C_{2}}^{\beta \alpha}(1,1)$.

(Vertices of $L_{C_{2}}^{\beta \alpha}(1,1)$ are indexed by $C_{2}$-semistandard tableaux.)
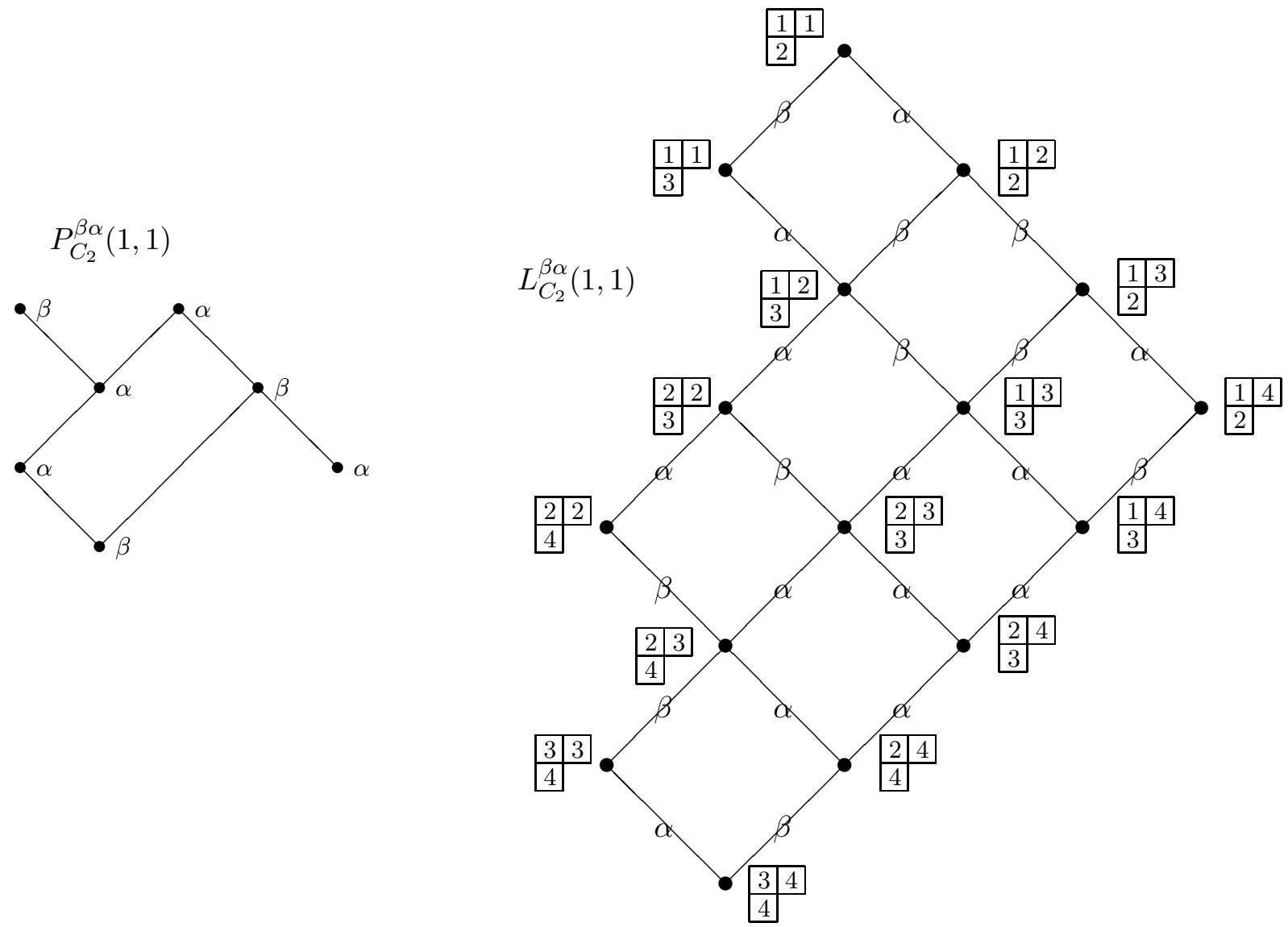

Figure 6.2. And $P_{1}$ and $P_{2}$ look similar in structure to the fundamental $\mathfrak{g}$-semistandard posets presented in Figure 4.2. But it can be seen that $L$ does not satisfy the structure condition for any $2 \times 2$ matrix $M$. Therefore $L$ cannot be a splitting poset for a representation, and so there is no hope of applying Corollary 5.4 to $L$. But $L$ does have a "symmetric chain decomposition," and hence it is rank symmetric, rank unimodal, and "strongly Sperner".

It is possible to prove that the $\mathfrak{g}$-semistandard lattices, $\mathfrak{g} \in\left\{A_{1} \oplus A_{1}, A_{2}, C_{2}, G_{2}\right\}$, are the only lattices of the kind we have been considering which can have the $M$-structure property for any $2 \times 2$ integer matrix $M$ :

Theorem 6.1 [Don2] Let $P$ be a two-color grid poset which has the max property. If $L=J_{\text {color }}(P)$ has the $M$-structure property for some $2 \times 2$ integer matrix $M$, then $L$ is a $\mathfrak{g}$-semistandard lattice, $\mathfrak{g} \in\left\{A_{1} \oplus A_{1}, A_{2}, C_{2}, G_{2}\right\}$.

Theorem 6.2 [Don2] Let $P$ be an indecomposable two-color grid poset. If $L=J_{\text {color }}(P)$ has the $M$-structure property for some $2 \times 2$ integer matrix $M$, then $L$ is a $\mathfrak{g}$-fundamental lattice, $\mathfrak{g} \in\left\{A_{1} \oplus A_{1}, A_{2}, C_{2}, G_{2}\right\}$.

These two statements are combinatorial Dynkin diagram classification theorems: No Lie theory or algebraic concepts of any kind appear in their hypotheses, but the short list of Dynkin diagramindexed rank two Cartan matrices plays the central role in their conclusions. 
Figure 6.2: We can write $P=Q_{1} \triangleleft Q_{2}$ with $Q_{i} \cong P_{i}(i=1,2)$. Below, $L=J_{\text {color }}(P)$.
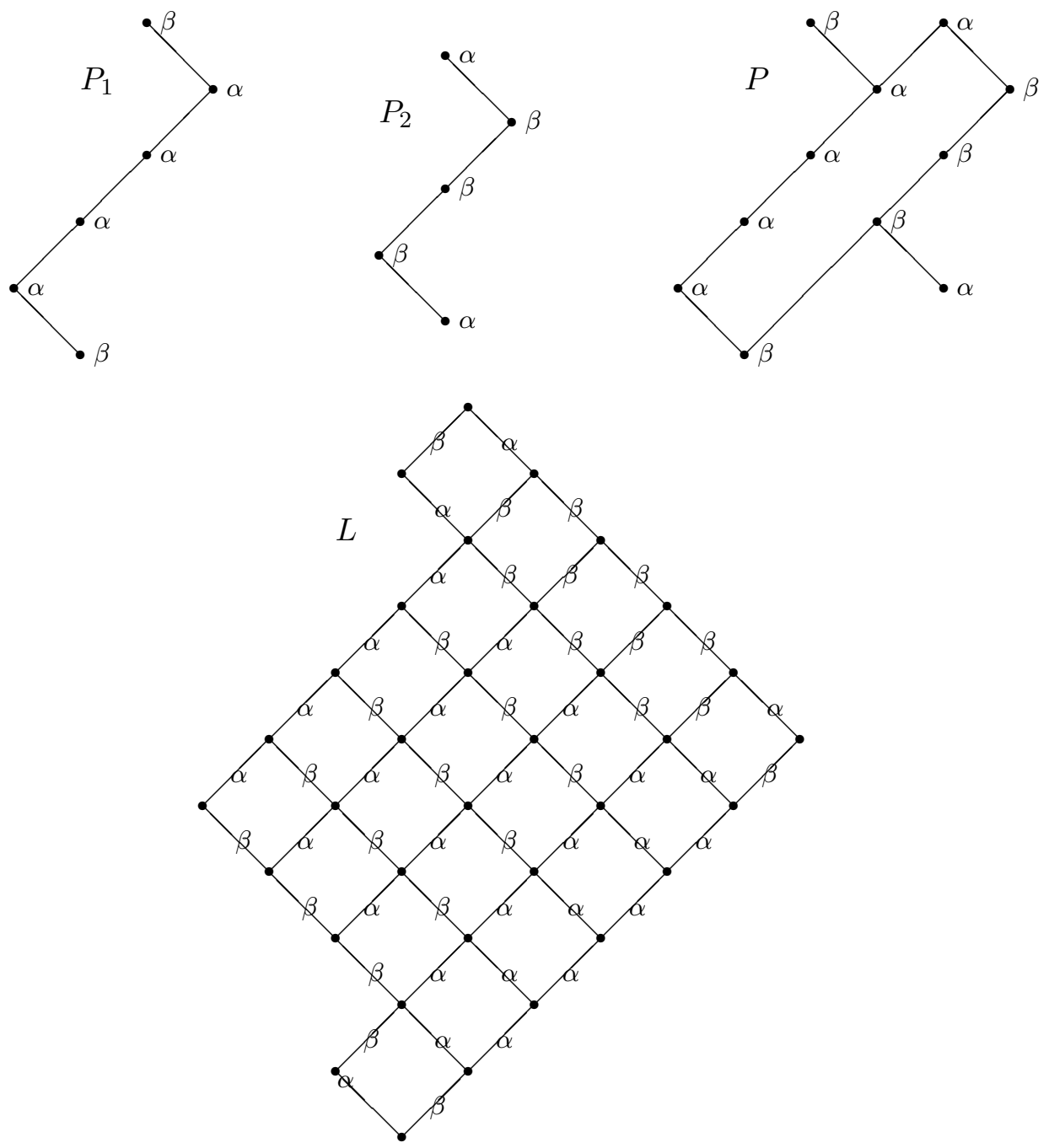

Let $P$ be a two-color grid poset and set $L=J_{\text {color }}(P)$. To apply Corollary 5.4 to $L$ via Theorem 5.3 , Proposition 5.2 was required: the elements of the $\mathfrak{g}$-semistandard lattices matched up with tableaux of Littelmann. The precise match-up required here should make one pessimistic about obtaining the rank generating function identities of Corollary 5.4 for these more general $L$. This pessimism is intuitively heightened by the classification results above, which emphasize how special the $\mathfrak{g}$-semistandard lattices are. (But it is possible to obtain the total count results mentioned at the end of Corollary 5.4 with elementary combinatorial reasoning [ADLP.) After representations for the cases listed in the introduction to this paper are constructed, Corollary 5.3 of [ADLP notes that the $\mathfrak{g}$-semistandard lattices in those cases are strongly Sperner. Although this approach cannot be used for the rest of the $\mathfrak{g}$-semistandard lattices, it is natural to hope that those lattices have this property. When addressing these extremal set theory issues, here it would now seem reasonable to attempt a combinatorial approach: Can one find symmetric chain decompositions of $L=J_{\text {color }}(P)$ for certain two-color grid posets $P$ ? 
Although the rank two cases in Lie theory are much simpler than the general rank cases, it is also true in Lie theory that the key aspect of a higher rank case often reduces to consideration of that aspect for just the rank two cases. Various aspects of this rank two paper will be used for many higher rank cases in [DW]. The forms of the $\mathfrak{g}$-semistandard tableaux of Section 4 may seem unmotivated to readers who are familiar only with [Hum]. Space and time permitting, much motivation could be supplied. Strict columns of length two arise because the second fundamental representation in each simple case can be realized as the "big piece" of the second exterior power of the first fundamental representation. Standard monomial theory (and earlier papers concerning algebras with straightening laws) explain how the restricted concatenation of columns corresponds to the multiplication of "Plücker coordinates" for flag manifolds. Going further, it may be possible to "explain" the simple root colorings of the elements of the posets $P$ in the spirit of the heaps of Stembridge, along the lines of Theorem 11.1 of [Pr1].

Our main result states that the $\mathfrak{g}$-semistandard lattices are splitting posets for their representations. For any representation, the crystal graph (of Kashiwara) is a splitting poset (cf. Lemma 3.6 of [Don1]). More generally, this is true for Stembridge's overarching crystal graph-like "admissible systems" [Stem]. The second author has observed that any such "general crystal graph" for a given representation is "edge minimal" within the set of splitting posets for the representation: It contains no splitting poset for the representation as a proper subgraph. For all irreducible representations of types $A_{2}$ and $C_{2}$, it can be seen from $[\mathrm{KN}$ that Kashiwara's crystal graphs are subgraphs of the corresponding $\mathfrak{g}$-semistandard lattices. It seems likely that all $\mathfrak{g}$-semistandard lattices give rise to admissible systems. This would directly show that the elements of these lattices generate the associated Weyl characters. In [DW] we will consider most simple Lie algebras of arbitrary rank and uniformly define $\mathfrak{g}$-fundamental posets for their fundamental weights which have the following property: the longest element in the associated Bruhat order is "fully commutative". This definition is type-independent. Using these fundamental posets, as in Section 4 we build $\mathfrak{g}$-semistandard posets and lattices for many representations. Along with this paper, this should start a new program: Find modular lattice splitting posets for all irreducible representations of all semisimple Lie algebras and show that they give rise to admissible systems. If these hopes are realized, these modular lattices (including the $\mathfrak{g}$-semistandard lattices) would in general contain "extra" edges with respect to the admissible system. But the lattices might be more combinatorially interesting than most or all admissible systems' directed graphs, and hopefully more accessible. One consequence might be the formulation of analogs of the Littlewood-Richardson tensor product rule in terms of manipulations of the underlying $\mathfrak{g}$-semistandard posets (or their analogs in the modular/non-distributive cases).

\section{References}

[Alv] L. W. Alverson II, "Distributive lattices and representations of the rank two simple Lie algebras," Master's thesis, Murray State University, 2003.

[ADLP] L. W. Alverson II, R. G. Donnelly, S. J. Lewis, and R. Pervine, "Constructions of representations of rank two semisimple Lie algebras with distributive lattices," Electronic J. Combin. , 13 (2006), \#R109 (44 pp). 
[Don1] R. G. Donnelly, "Extremal properties of bases for representations of semisimple Lie algebras," $J$. Algebraic Comb. 17 (2003), 255-282.

[Don2] R. G. Donnelly, "A Dynkin diagram classification of posets satisfying certain structural properties," preprint.

[DLP] R. G. Donnelly, S. J. Lewis, and R. Pervine, "Constructions of representations of $\mathfrak{o}(2 n+1, \mathbb{C})$ that imply Molev and Reiner-Stanton lattices are strongly Sperner," Discrete Math. 263 (2003), $61-79$.

[DW] R. G. Donnelly and N. J. Wildberger, "Distributive lattice models for certain families of irreducible semisimple Lie algebra representations," in preparation.

[Hum] J. E. Humphreys, Introduction to Lie Algebras and Representation Theory, Springer, New York, 1972 .

[KN] M. Kashiwara and T. Nakashima, "Crystal graphs for representations of the $q$-analogue of classical Lie algebras," J. Algebra 165 (1994), 295-345.

[Lit] P. Littelmann, "A generalization of the Littlewood-Richardson rule," J. Algebra 130 (1990), 328-368.

[Mc] M. McClard, "Picturing Representations of Simple Lie Algebras of Rank Two," Master's thesis, Murray State University, 2000.

[Pr1] R. A. Proctor, "Bruhat lattices, plane partition generating functions, and minuscule representations," European J. Combin. 5 (1984), 331-350.

[Pr2] R. A. Proctor, "Solution of a Sperner conjecture of Stanley with a construction of Gelfand," $J$. Comb. Th. A 54 (1990), 225-234.

[Sta1] R. P. Stanley, "Unimodal sequences arising from Lie algebras," in: Young Day Proceedings, ed. T. V. Narayana et al, Marcel Dekker, New York, 1980, 127-136.

[Sta2] R. P. Stanley, Enumerative Combinatorics, Vol. 1, Wadsworth and Brooks/Cole, Monterey, CA, 1986.

[Sta3] R. P. Stanley, Enumerative Combinatorics, Vol. 2, Cambridge University Press, 1999.

[Stem] J. Stembridge, "Combinatorial models for Weyl characters," Advances in Math. 168 (2002), 96-131. 